\title{
Cortical astrocytes rewire somatosensory cortical circuits for peripheral neuropathic pain
}

\author{
Sun Kwang Kim, ${ }^{1,2,3}$ Hideaki Hayashi, ${ }^{3,4}$ Tatsuya Ishikawa, ${ }^{2,3,5}$ Keisuke Shibata, ${ }^{3,4}$ Eiji Shigetomi, ${ }^{3,4}$ Youichi Shinozaki, ${ }^{3,4}$ \\ Hiroyuki Inada, ${ }^{2,3}$ Seung Eon Roh, ${ }^{6}$ Sang Jeong Kim, ${ }^{6}$ Gihyun Lee, ${ }^{1}$ Hyunsu Bae, ${ }^{1}$ Andrew J. Moorhouse, ${ }^{7}$ \\ Katsuhiko Mikoshiba, ${ }^{8}$ Yugo Fukazawa, ${ }^{3,9}$ Schuichi Koizumi, ${ }^{3,4}$ and Junichi Nabekura, ${ }^{2,3,5}$ \\ 1Department of Physiology, College of Korean Medicine, Kyung Hee University, Seoul, South Korea. ²Division of Homeostatic Development, National Institute for Physiological Sciences, Okazaki, Japan. \\ ${ }^{3}$ Core Research for Evolutional Science and Technology, Japan Science and Technology Agency, Saitama, Japan. ${ }^{4}$ Department of Neuropharmacology, Interdisciplinary Graduate School of Medicine, University \\ of Yamanashi, Yamanashi, Japan. 들. \\ Seoul, South Korea. ' School of Medical Sciences, University of New South Wales, Sydney, Australia. ${ }^{8}$ Laboratory for Developmental Neurobiology, Brain Science Institute, RIKEN, Saitama, Japan. \\ ${ }^{9}$ Division of Cell Biology and Neuroscience, Department of Histological and Physiological Sciences, Faculty of Medical Science, University of Fukui, Fukui, Japan.
}

\begin{abstract}
Long-term treatments to ameliorate peripheral neuropathic pain that includes mechanical allodynia are limited. While glial activation and altered nociceptive transmission within the spinal cord are associated with the pathogenesis of mechanical allodynia, changes in cortical circuits also accompany peripheral nerve injury and may represent additional therapeutic targets. Dendritic spine plasticity in the $\mathbf{S 1}$ cortex appears within days following nerve injury; however, the underlying cellular mechanisms of this plasticity and whether it has a causal relationship to allodynia remain unsolved. Furthermore, it is not known whether glial activation occurs within the S1 cortex following injury or whether it contributes to this S1 synaptic plasticity. Using in vivo 2-photon imaging with genetic and pharmacological manipulations of murine models, we have shown that sciatic nerve ligation induces a re-emergence of immature metabotropic glutamate receptor 5 (mGluR5) signaling in S1 astroglia, which elicits spontaneous somatic $\mathrm{Ca}^{2+}$ transients, synaptogenic thrombospondin 1 (TSP-1) release, and synapse formation. This $\mathrm{S} 1$ astrocyte reactivation was evident only during the first week after injury and correlated with the temporal changes in S1 extracellular glutamate levels and dendritic spine turnover. Blocking the astrocytic mGluR5-signaling pathway suppressed mechanical allodynia, while activating this pathway in the absence of any peripheral injury induced long-lasting (>1 month) allodynia. We conclude that reawakened astrocytes are a key trigger for S1 circuit rewiring and that this contributes to neuropathic mechanical allodynia.
\end{abstract}

\section{Introduction}

Chronic pain and long-term memory share very similar mechanisms that involve neural circuit remodeling and synaptic plasticity as a key model $(1,2)$. The formation of new synaptic connections in the adult cortex underlies the transition from short-term to long-term memory $(3,4)$. Such circuit and synaptic plasticity has been documented in a number of cortical regions associated with nociception including the medial prefrontal cortex $(5,6)$, the anterior cingulate cortex $(7,8)$ and the primary somatosensory (S1) cortex $(9,10)$, and these changes may be required for a shift from acute to chronic pain.

The excitatory neural circuits in the $\mathrm{S} 1$ cortex primarily process behaviorally relevant somatosensory information such as the location and intensity of touch or pain $(11,12)$. Direct manipulation of the $\mathrm{S} 1$ cortex modulates the neuronal activity evoked by innocuous and noxious peripheral stimulation in downstream brain areas associated with pain signaling including the thalamus and anterior cingulate cortex $(9,13)$. Such S1 cortex manipulation can temporarily relieve neuropathic pain in humans and animals (14-16). This

Conflict of interest: The authors have declared that no conflict of interest exists. Submitted: May 18, 2015; Accepted: February 25, 2016.

Reference information: J Clin Invest. 2016;126(5):1983-1997. doi:10.1172/JCI82859. suggests that the S1 cortex might play a role as a "central processing unit" within the brain networks that mediate and/or sustain chronic neuropathic pain. Our recent studies $(10,17)$ on S1 postsynaptic dendritic spines, on which the majority of excitatory inputs synapse (18), demonstrated an acute and transient increase in spine turnover following peripheral nerve injury. Specifically, new spines were markedly formed in the early post-injury phase ( 1 week), and these became persistent and substantially larger during the later postinjury phase ( 2 weeks). In contrast, new spines that were generated before nerve injury became smaller or disappeared altogether following injury (10). These structural synaptic changes were associated with both an increased S1 cortex excitability and mechanical allodynia. These observations led to the proposal that the early and transient increase in S1 spine turnover might be important for the chronic changes in neuropathic pain behavior, akin to the dendritic spine plasticity in learning and memory $(10,19,20)$. To evaluate and realize the potential of such $\mathrm{S} 1$ structural synaptic remodeling as a novel therapeutic target for neuropathic mechanical allodynia, it is critical to first determine whether these structural changes may be causative for mechanical allodynia and, second, to elucidate the underlying cellular mechanisms of such S1 cortex plasticity.

Glial cells are known to make important contributions to functional and structural neuronal plasticity. Astrocytes, a major type 
of glia, respond to neuronal activity with increases in intracellular $\mathrm{Ca}^{2+}(21,22)$ and can directly influence synaptic connectivity by releasing synaptogenic molecules (23). This is particularly important in the developing nervous system when extensive neural circuit remodeling occurs. Hence, we hypothesize that S1 astrocytes may show functional changes following peripheral nerve injury that induces a modification of synaptic connections, resulting in mechanical hypersensitivity in neuropathic pain. Our results implicate a causal role of cortical astrocytes and S1 structural synaptic plasticity in mechanical allodynia and point to potential new targets for the mitigation of debilitating allodynia.

\section{Results}

Somatic $\mathrm{Ca}^{2+}$ transients in contralateral $\mathrm{S} 1$ cortex astrocytes following peripheral nerve injury contribute to mechanical allodynia. Using in vivo 2-photon microscopic imaging, we examined basal somatic $\mathrm{Ca}^{2+}$ activity in astrocytes in the contralateral (left) S1 cortex of living adult mice following a partial ligation of the right sciatic nerve (PSL injury) (Figure 1, A and B). The frequency of basal astrocytic $\mathrm{Ca}^{2+}$ transients (defined as a change in fluorescence intensity relative to baseline $\left.\left[\Delta \mathrm{F} / \mathrm{F}_{0}\right]>15 \%\right)$ and the proportion of "active astrocytes" that showed 1 or more $\mathrm{Ca}^{2+}$ transients during the 10 -minute recording period were markedly increased when measured during the first week after injury (3-6 days), before partially returning to baseline approximately 2 weeks (12-15 days) after injury (Figure 1, B-D, and Supplemental Videos 1 and 2; supplemental material available online with this article; doi:10.1172/JCI82859DS1). The amplitude of $\mathrm{Ca}^{2+}$ transients was unchanged during both of these phases (Figure 1D), suggesting that there was an increase in the induction of $\mathrm{S} 1$ astrocytic $\mathrm{Ca}^{2+}$ transients rather than an increase in the extent of intracellular $\mathrm{Ca}^{2+}$. The increase in $\mathrm{Ca}^{2+}$ transients of $\mathrm{S} 1$ astrocytes was temporarily well matched to both the increase in S1 dendritic spine turnover and to the period during which mechanical allodynia develops, all of which occur during the first week after injury (ref. 10 and see also Figure 1C). We also quantified the S1 synaptic density, i.e., the number of colocalized presynaptic VGlut1 and postsynaptic PSD-95 puncta (24), in sham control and PSL injury groups. The results showed that synaptic density in the PSL group was significantly higher than that in the sham group $(165.7 \%$ $\pm 11.3 \%$ increase in the PSL group, normalized to sham, Figure 1E). This increase in synaptic density is likely due to a marked increase in PSD-95 (154.3\% $\pm 8.1 \%$ increase, normalized to sham) and a mild increase in VGlut1 puncta $(120.8 \% \pm 8.0 \%$ increase, normalized to sham), which matches well with our previous data showing a strong increase in dendritic spines (10) and a modest increase in axonal boutons (17) following PSL injury. These results indicate that the observed dendritic spine changes may contribute to the increase in new synapse formation after PSL injury.

To examine the behavioral consequences of the increased astrocytic $\mathrm{Ca}^{2+}$ activity, we used inositol-1,4,5-trisphosphate receptor type 2-KO (Itpr $2^{-/-}$, herein referred to as $\left.\mathrm{IP}_{3} \mathrm{R} 2-\mathrm{KO}\right)$ mice (25). $\mathrm{IP}_{3} \mathrm{R} 2$ is selectively expressed in astrocytes, and astrocyte somatic $\mathrm{Ca}^{2+}$ transients are absent in these mice, while $\mathrm{IP}_{3} \mathrm{R} 2$ is not expressed in neurons, and neuronal $\mathrm{Ca}^{2+}$ transients are unaffected (26-28). We also confirmed a lack of S1 astrocytic $\mathrm{Ca}^{2+}$ transients following PSL in $\mathrm{IP}_{3} \mathrm{R} 2-\mathrm{KO}$ mice (Supplemental Figure $1 \mathrm{~A})$, while frequent $\mathrm{Ca}^{2+}$ transients were still observed in S1 neu- rons (Supplemental Video 3). Mechanical allodynia for up to at least 1 month following PSL injury was significantly reduced in $\mathrm{IP}_{3} \mathrm{R} 2-\mathrm{KO}$ mice (Figure $1 \mathrm{~F}$ ). There were no significant differences in motor function, as assessed by the rotarod test, between WT and $\mathrm{IP}_{3} \mathrm{R} 2-\mathrm{KO}$ mice, either before or after PSL injury (Supplemental Figure 1B). Somatosensory-evoked potentials recorded in the S1 cortex were increased following PSL injury (Supplemental Figure 1C), as observed in our previous study (10), and such enhanced S1 neuronal excitability was absent in IP 32 2-KO mice (Supplemental Figure 1C). Topical infusion of 1,2-bis(o-aminophenoxy)ethane-N,N,N',N'-tetraacetic acid (BAPTA-AM, a $\mathrm{Ca}^{2+}$ chelator) into the cortical surface can also be used to selectively inhibit astrocytic $\mathrm{Ca}^{2+}$ transients (29), as, in the intact cortex, the AM-based $\mathrm{Ca}^{2+}$ indicators selectively load into astrocytes but not neurons $(28,30)$. To apply BAPTA-AM less invasively over a prolonged period, we used the noninflammatory, sustained ( 6 days) Elvax drug delivery system (for the specificity of Elvax drug delivery, see Supplemental Figure 1, D and E). Such local S1 infusion of BAPTA-AM blocked the increase in astrocytic $\mathrm{Ca}^{2+}$ transients following PSL injury (Supplemental Figure 2A), without changing neuronal $\mathrm{Ca}^{2+}$ activity (Supplemental Figure 2B). Elvax-mediated infusion of BAPTA-AM also significantly attenuated mechanical allodynia for at least 1 month (Figure $1 \mathrm{~F}$ ). Similarly, Elvax-mediated infusion of an astrocyte metabolism inhibitor, fluoroacetate, which also selectively suppressed astrocytic $\mathrm{Ca}^{2+}$ transients (data not shown), alleviated mechanical allodynia (Figure 1F). Thus, both genetic and pharmacological approaches demonstrate that spontaneous astrocyte somatic $\mathrm{Ca}^{2+}$ transients in the S1 cortex emerge in the first week following PSL injury, and preventing these $\mathrm{Ca}^{2+}$ transients markedly reduces chronic mechanical allodynia.

Manipulation of somatic $\mathrm{Ca}^{2+}$ transients in S1 astrocytes modulates structural synaptic plasticity. The peak in spontaneous S1 astrocytic $\mathrm{Ca}^{2+}$ transients in the first week after PSL injury coincides with the peak in synapse remodeling following PSL (as measured by spine turnover; Figure 1C). This suggests a possible causal relationship between the two. Thus, we investigated the effects of stimulating astrocytic $\mathrm{Ca}^{2+}$ transients in naive mice (Figure $2 \mathrm{~A}$ ) using repeated in vivo 2-photon photolysis of caged $\mathrm{Ca}^{2+}$ 1-(4,5-dimethoxy-2nitrophenyl)-1,2-diaminoethane-N,N,N',N'-tetraacetic acid (DMNPEDTA). We used the thy1GFP-M mouse line, as these mice express EGFP in a small subset of layer V pyramidal neurons (31), enabling easy identification of dendritic spines. Our protocol involved evoking somatic $\mathrm{Ca}^{2+}$ elevations every 5 minutes, while repeatedly imaging the same apical dendrites every 30 minutes (Figure 2B and see also Methods). This modest frequency of evoked somatic $\mathrm{Ca}^{2+}$ transients in $\mathrm{S} 1$ astrocytes was still sufficient to induce an acute and significant increase in spine turnover (formation and retraction/elimination) in dendritic segments adjacent to the stimulated astrocytes as compared with that observed in remote dendritic segments (Figure 2C and see also Supplemental Figure 3 for quantification).

In the converse experiment, we repeatedly imaged the same dendrites in S1 cortex before and 3 days after PSL injury and compared the extent of spine turnover in control mice and in mice that received Elvax-mediated local BAPTA-AM infusion (Figure 3A and see also Methods). As we previously reported (10), and as shown in Figure 1C, PSL injury induced a rapid increase in the rate of spine 
A

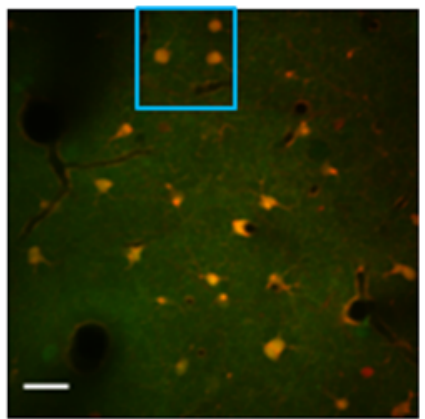

B

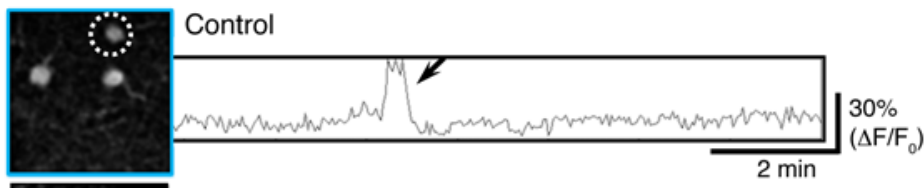

C

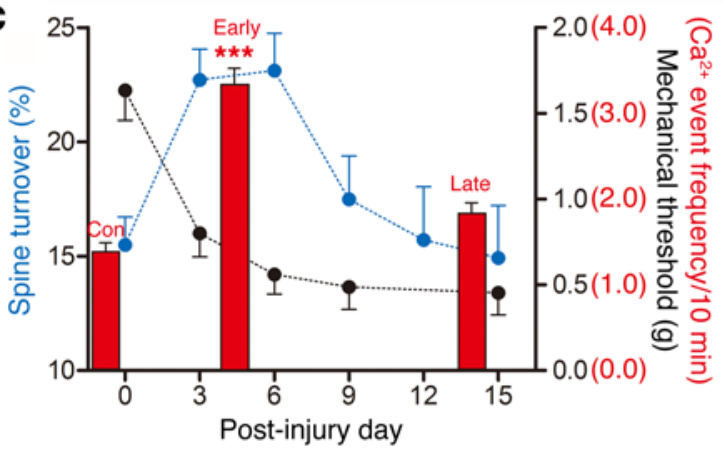

D

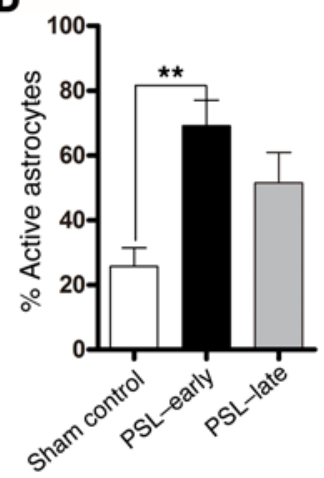

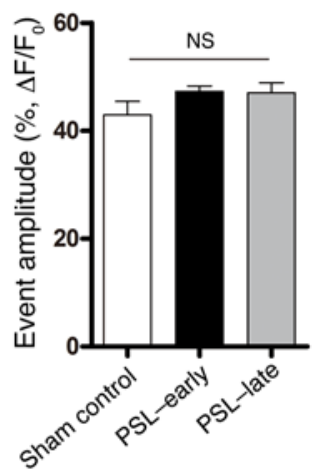

E
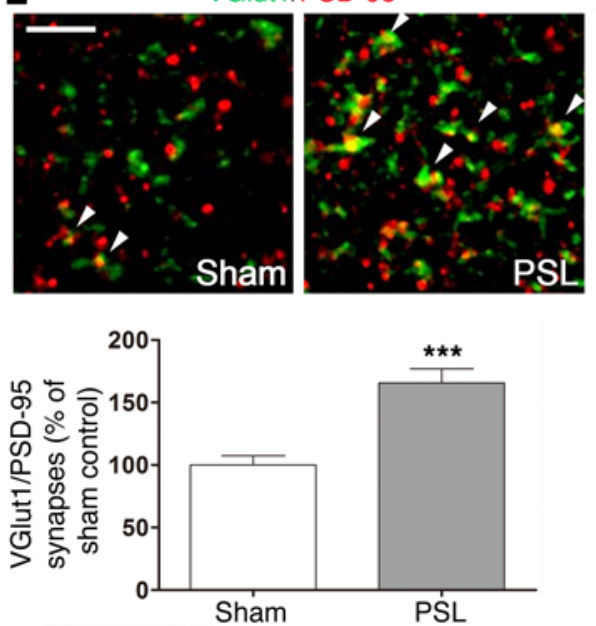

$\mathbf{F}$

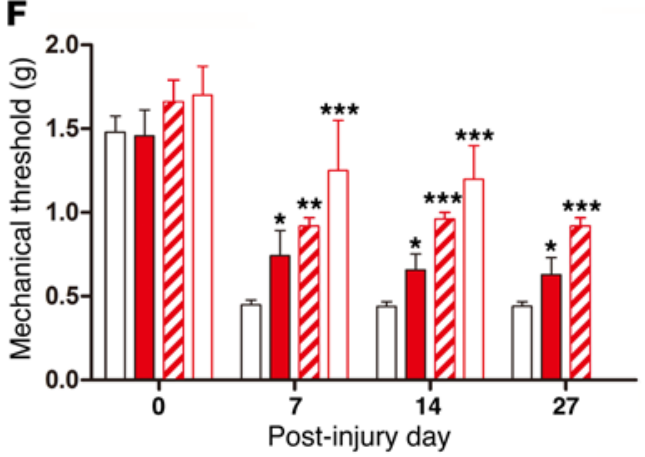

$\square$ Control IP ${ }_{3}$ R2-KO $\square$ Fluoroacetate BAPTA-AM / Fluoroacetate

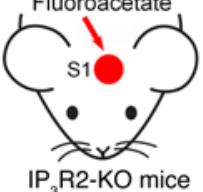

$\mathrm{IP}_{3} \mathrm{R} 2-\mathrm{KO}$ mice 2-photon $\mathrm{Ca}^{2+}$ imaging of astrocytes (yellow staining) in S1 cortex layer I, loaded with OCB-1-AM (Ca ${ }^{2+}$ indicator, green) and SR101 (astrocyte marker, red). Scale bar: $30 \mu \mathrm{m}$. (B) Representative images of $\mathrm{S} 1$ astrocytes (dashed circles) and corresponding $\mathrm{Ca}^{2+}$ transients in control (blue rectangle shown in A), PSL-early (3-6 days after PSL injury) and PSL-late (12-15 days after PSL injury) groups. Arrows indicate individual transients $\left(\Delta F / F_{0}>15 \%\right)$. (C) Frequency of astrocytic $\mathrm{Ca}^{2+}$ transients (red), spine turnover rate ( $n=550$ spines $/ 15$ dendrites $/ 6$ mice, blue), and mechanical threshold ( $n=6$ mice, black). Spine turnover and mechanical threshold data are from our previous study (10) conducted under the same conditions, with additional data from 1 mouse included. Ca ${ }^{2+}$ transients were quantified in active astrocytes. Con, sham-operated mice ( $n=41$ cells $/ 3$ mice); Early ( $n=130$ cells $/ 3$ mice); Late ( $n=71$ cells $/ 4$ mice). ${ }^{* * *} P<0.001$ versus control, by Kruskal-Wallis test. (D) Left: Proportion of active astrocytes that showed 1 or more Ca ${ }^{2+}$ events during a 10-minute recording period in sham control, PSL-early, and PSL-late mice. $n=10-13$ imaged planes/group. ${ }^{*} P<0.01$, by Kruskal-Wallis test. Right: Corresponding mean amplitudes of $\mathrm{Ca}^{2+}$ transients. NS, $P>0.05$, by Kruskal-Wallis test. (E) Top: Representative confocal images of synapses (arrowheads) indicated by colocalization of presynaptic (VGlut1, green) and postsynaptic (PSD-95, red) markers in the sham and PSL injury groups. Scale bar: $5 \mu \mathrm{m}$. Bottom: Quantitative analysis demonstrates that PSL injury significantly increased the number of colocalized synaptic puncta. $n=12$ image stacks/4 mice per group. ${ }^{* *} P<0.001$ versus sham control, by unpaired $t$ test. (F) Mean mechanical thresholds $0-27$ days following PSL injury in control $(n=10)$, IP $R 2-K O(7)$, BAPTA-AM (5), and fluoroacetate-treated (4) mice. ${ }^{*} P<0.05,{ }^{*} P<0.01$, and ${ }^{* * *} P<0.001$ versus control, by 1 -way ANOVA. Error bars represent the mean \pm SEM. 
A Before
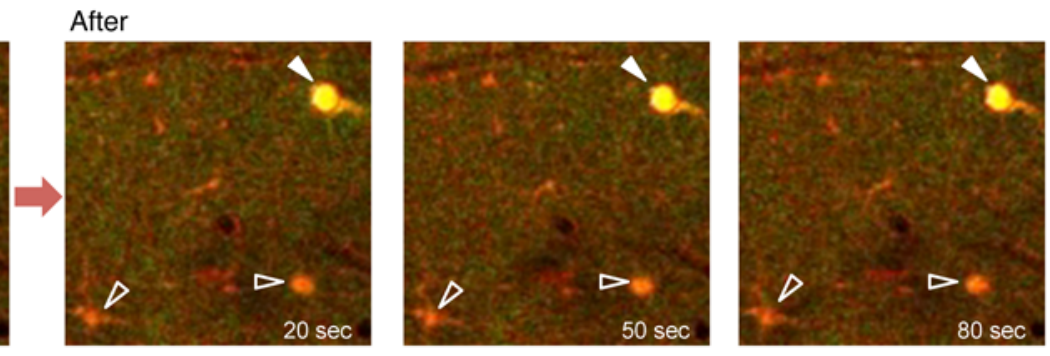

B
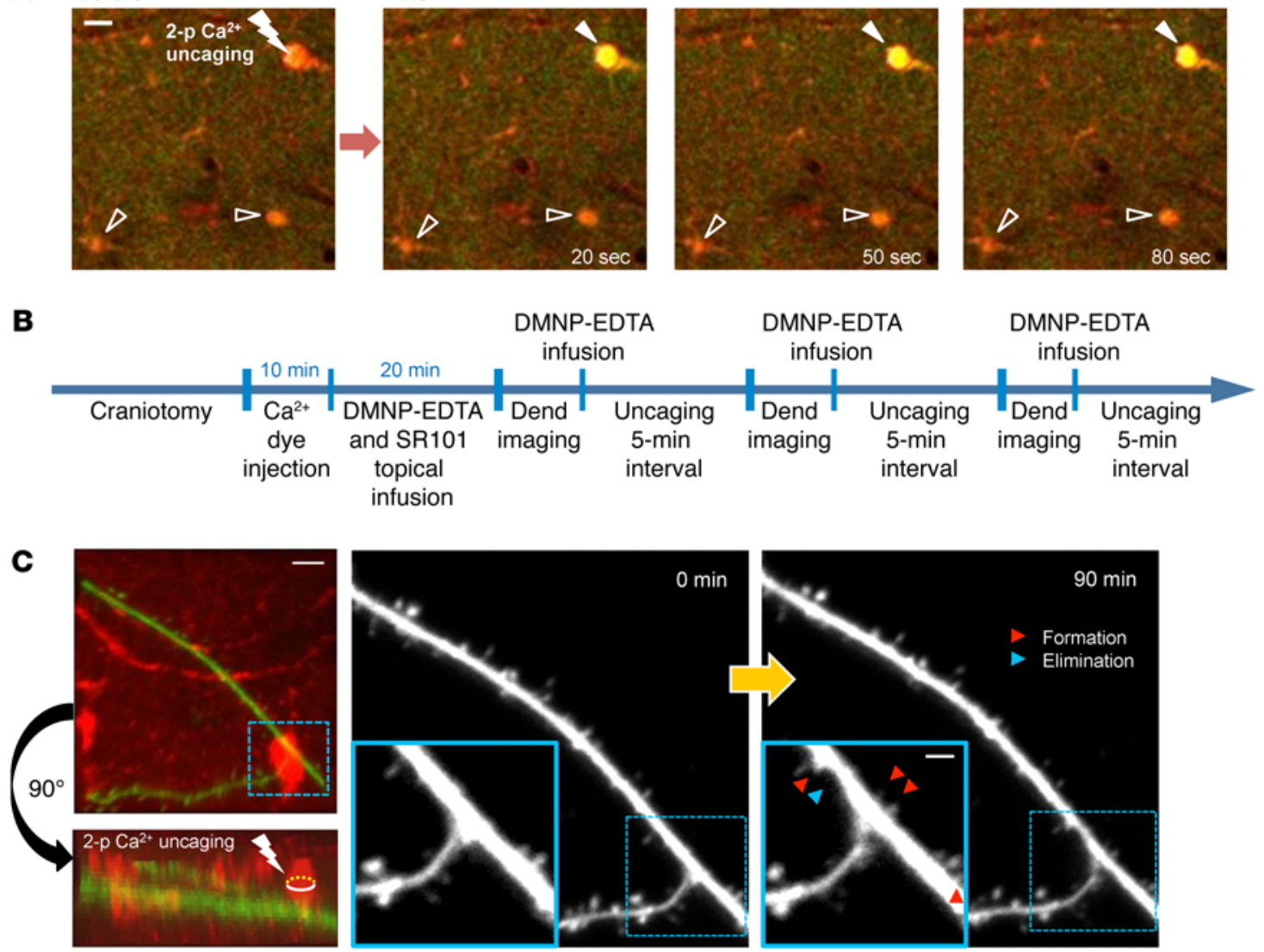

Figure 2. Activation of $\mathbf{S 1}$ astrocytic $\mathrm{Ca}^{2+}$ induces spine turnover. (A) Photolysis of caged $\mathrm{Ca}^{2+}$ (DMNP-EDTA) within an astrocyte soma was done by 730 $\mathrm{nm} 2$-photon laser stimulation ( $10 \mathrm{~mW}, 50$-ms duration, Tornado mode, Olympus). This brief uncaging induced a prolonged ( $\geq 1$ minute) astrocytic Ca ${ }^{2+}$ transient (solid arrowheads; yellow) approximately 20 seconds after the stimulation. Simultaneous imaging (0.5 Hz, 900 -nm 2-photon laser scanning) was done after dye loading ( $\mathrm{Ca}^{2+}$ dye [OCB-1-AM, green] and astrocyte marker [SR101, red]) in a naive mouse. Note that the unstimulated astrocytes (open arrowheads) show no $\mathrm{Ca}^{2+}$ changes. Scale bar: $10 \mu \mathrm{m}$. (B) Timeline of the experimental protocol for in vivo 2-photon Ca ${ }^{2+}$ uncaging (from DMNP-EDTA) and repeated dendrite (Dend) imaging. (C) Left: Z-projection images of apical dendrites (green, GFP) and astrocytes (red, SR101). Scale bar: $10 \mu \mathrm{m}$. Yellow oval in lower rotated image indicates the focal point for in vivo intra-astrocyte 2-photon $\mathrm{Ca}^{2+}$ uncaging. Center and right: Magnified dendrite images before (0 minutes) and 90 minutes after $\mathrm{Ca}^{2+}$ uncaging. Repeated astrocytic $\mathrm{Ca}^{2+}$ uncaging (5-minute intervals) induced spine formation and elimination in adjacent dendritic segments (blue squares), but not in more remote dendritic segments (upper left of center and right images). Scale bar: $3 \mu \mathrm{m}$.

formation and elimination as compared with that seen in shamoperated controls, and this was also apparent when saline was topically applied (via Elvax) to the S1 cortex (Figure 3, B and C). In contrast, local application of BAPTA-AM to inhibit $\mathrm{S} 1$ astrocytic $\mathrm{Ca}^{2+}$ transients prevented this PSL injury-induced increase in the spine turnover rate (Figure 3, B and C). The effects of BAPTA-AM on spine elimination (not significant; $P>0.05$, PSL-BAPTA-AM vs. shamsaline or PSL-saline) was more modest than the apparent block of PSL injury-induced spine formation $(P<0.01$, PSL-BAPTA-AM vs. PSL-saline) (Figure 3C). Taken together, these results suggest that an increase in somatic $\mathrm{Ca}^{2+}$ transients in $\mathrm{S} 1$ astrocytes is sufficient and necessary to induce the structural synaptic plasticity in S1 cortical dendrites that occurs after peripheral nerve injury.

$\mathrm{Ca}^{2+}$-dependent release of TSP-1 from S1 cortex astrocytes following PSL injury induces synaptic rewiring and mechanical allodynia. We next sought to identify a potential molecular mechanism that causally links astrocytic $\mathrm{Ca}^{2+}$ activity, spine plasticity, and mechanical allodynia. Given that spine formation seemed more dependent on astrocytic $\mathrm{Ca}^{2+}$ signaling than did spine elimination
(Figure 3C), we focused on the thrombospondins 1 and 2 (TSP$1 / 2$ ), which are astrocyte-secreted soluble proteins that induce excitatory synapse formation during neuronal development or following cortical damage $(32,33)$. Indeed, TSP-1 levels in whole S1 cortex tissue extracts and in dialysates of S1 cortex extracellular fluid were both significantly increased in the early phase of PSL injury-induced mechanical allodynia, before returning to baseline levels during the late phase (Figure 4, A and B). Interestingly, this increase in TSP-1 was not seen in the IP ${ }_{3} \mathrm{R} 2-\mathrm{KO}$ mice (Figure $4 \mathrm{~A}$ ), indicating its dependence on astrocytic $\mathrm{Ca}^{2+}$ signaling. TSP-2 was not detected at either time point, nor was it detected in control conditions before PSL injury, whereas high levels of TSP-2 protein $(1,657.54 \pm 98.04 \mathrm{pg} / \mathrm{mg}$ protein, $n=4)$ were detected in the P8 mouse cortex that was used as a positive control (34). TSP-4 protein levels were not significantly increased after PSL injury (sham, $4.38 \pm 1.84$ vs. PSL, $5.88 \pm 1.79 \mathrm{ng} / \mathrm{mg}$ protein; $n=7-8 \mathrm{mice} /$ group; $P=0.28$, Mann-Whitney $U$ test).

FISH for Tsp1 mRNA combined with IHC for GFAP (astrocyte marker) showed that the expression levels of Tsp1 mRNA in astro- 
A
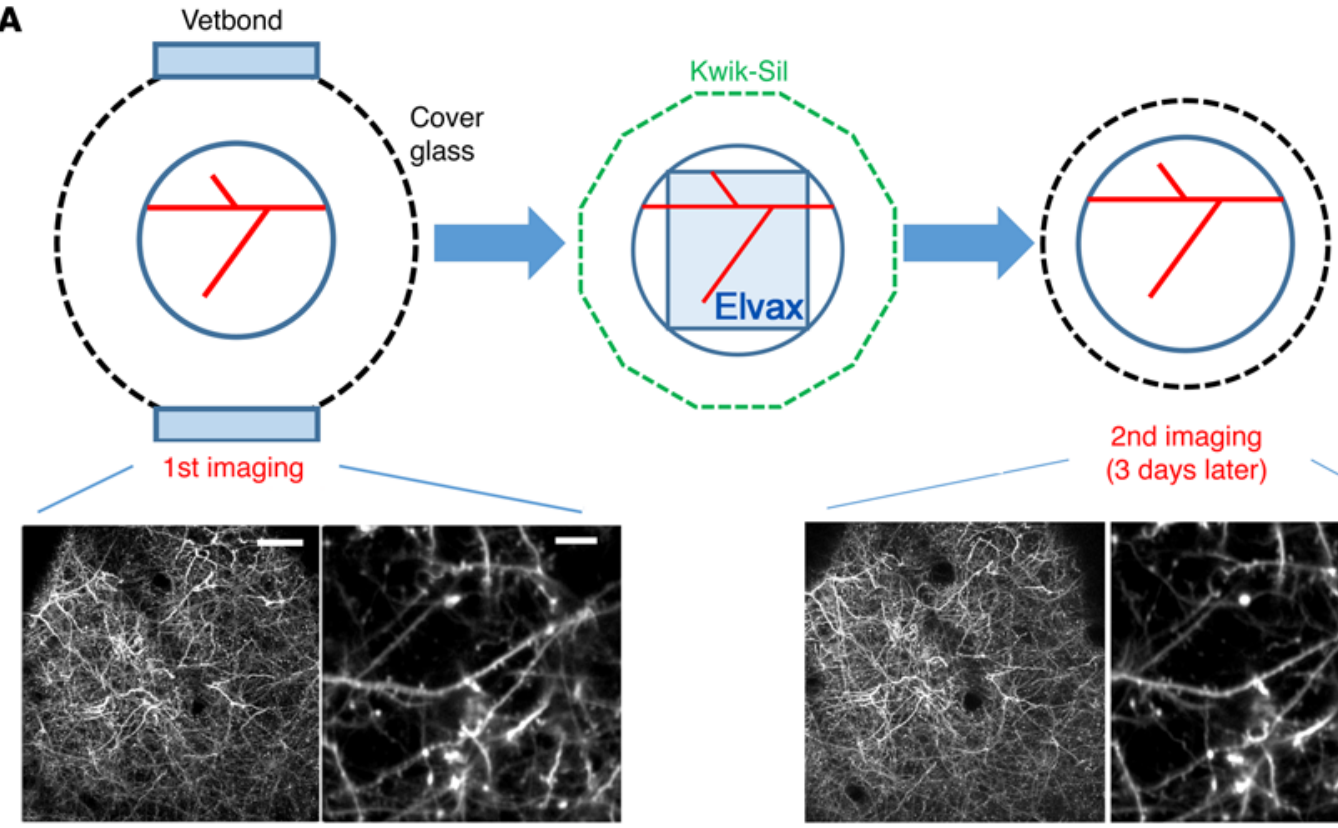

2nd imaging

( 3 days later)
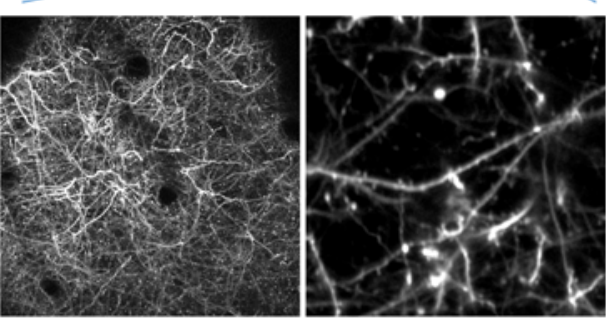

B

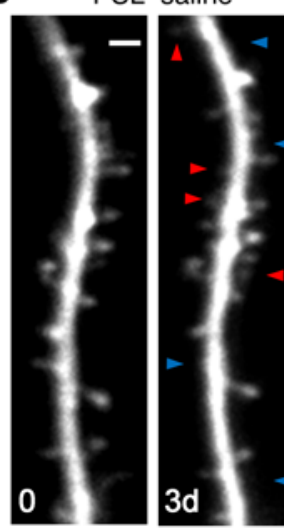

PSL-BAPTA-AM

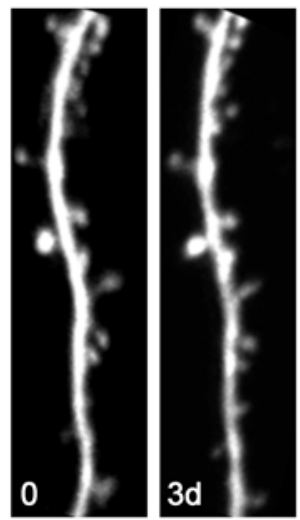

C

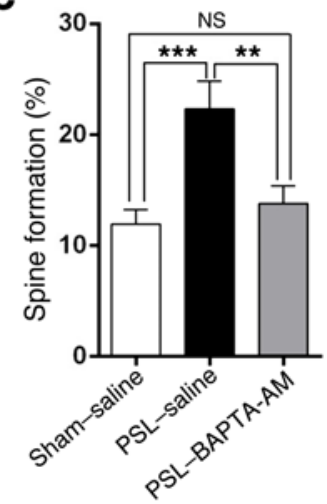

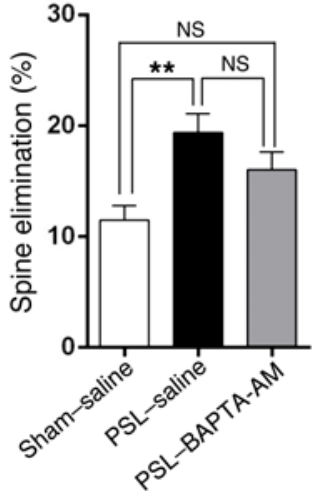

Figure 3. Inactivation of S1 astrocytic $\mathbf{C a}^{2+}$ inhibits spine turnover. (A) Repeated in vivo 2-photon imaging of the same dendrites before and 3 days after drug infusion into S1 cortex. Schematic procedure (top) and representative images (bottom) used for repeated imaging of the same apical dendrites of layer $\mathrm{V}$ pyramidal neurons in S1 cortex, before and 3 days after Elvax drug application. (B) Images of the same S1 cortex apical dendrite before and 3 days after PSL injury, with local sustained (Elvax) delivery of saline or BAPTA-AM. Arrowheads indicate spine formation (red) and elimination (blue). Scale bar: $2 \mu \mathrm{m}$. (C) Spine formation (left) and elimination (right) rates in saline sham-operated ( $n=24$ dendrites/3 mice), PSL-injured ( $n=17$ dendrites/3 mice), and BAPTA-AM PSL-injured groups ( $n=20$ dendrites $/ 3$ mice). ${ }^{* *} P<0.01$ and ${ }^{* * *} P<0.001$, by 1 -way ANOVA. Error bars represent the mean \pm SEM.

cytes $\left(\mathrm{GFAP}^{+}\right)$were significantly increased in the PSL injury group as compared with levels in the sham control group (Figure 4C). In contrast, Tsp $1 \mathrm{mRNA}$ expression levels in other cells (GFAP-) were not significantly different between the 2 groups (Figure $4 \mathrm{C}$ ). Immunofluorescence labeling also indicated that TSP-1 immunoreactivity in astrocytes, but not in other cells (GFAP-), was significantly increased following PSL injury (Figure 4D). Although a very recent study showed that microglia promote learning-related synaptogenesis via brain-derived neurotropic factor (BDNF) signaling (35), microglia showed no TSP-1 expression in our study (Supplemental Figure 4A). Our preliminary screen of the BDNF-signaling inhibitor K252a by infusion into $\mathrm{S} 1$ cortex showed no effect on mechanical allodynia. Moreover, microglial activation has not been observed in supraspinal regions following peripheral nerve injury (36).
Elvax-mediated infusion of the neuronal TSP receptor $\left(\alpha_{2} \delta-1\right)$ antagonist gabapentin (32) into the S1 cortex suppressed the increase in the spine formation rate after PSL injury (Figure 5A) and markedly attenuated mechanical allodynia 7, 14, and 27 days after injury (Figure 5B). Gabapentin did not affect the PSLinduced increase in spontaneous astrocytic $\mathrm{Ca}^{2+}$ transients (Supplemental Figure 4B), indicating that its action is downstream of astrocytic $\mathrm{Ca}^{2+}$. siRNA knockdown of TSP-1 expression in the S1 cortex also significantly inhibited PSL injury-induced spine formation (Figure 5C) and alleviated mechanical allodynia for at least 1 month (Figure 5B). As expected, both TSP-1 siRNA and gabapentin were less efficacious against the PSL injury-induced increase in the spine elimination rate (Figure 5, A and C). Remarkably, a single injection of TSP-1 into the S1 cortex of naive (nonin- 

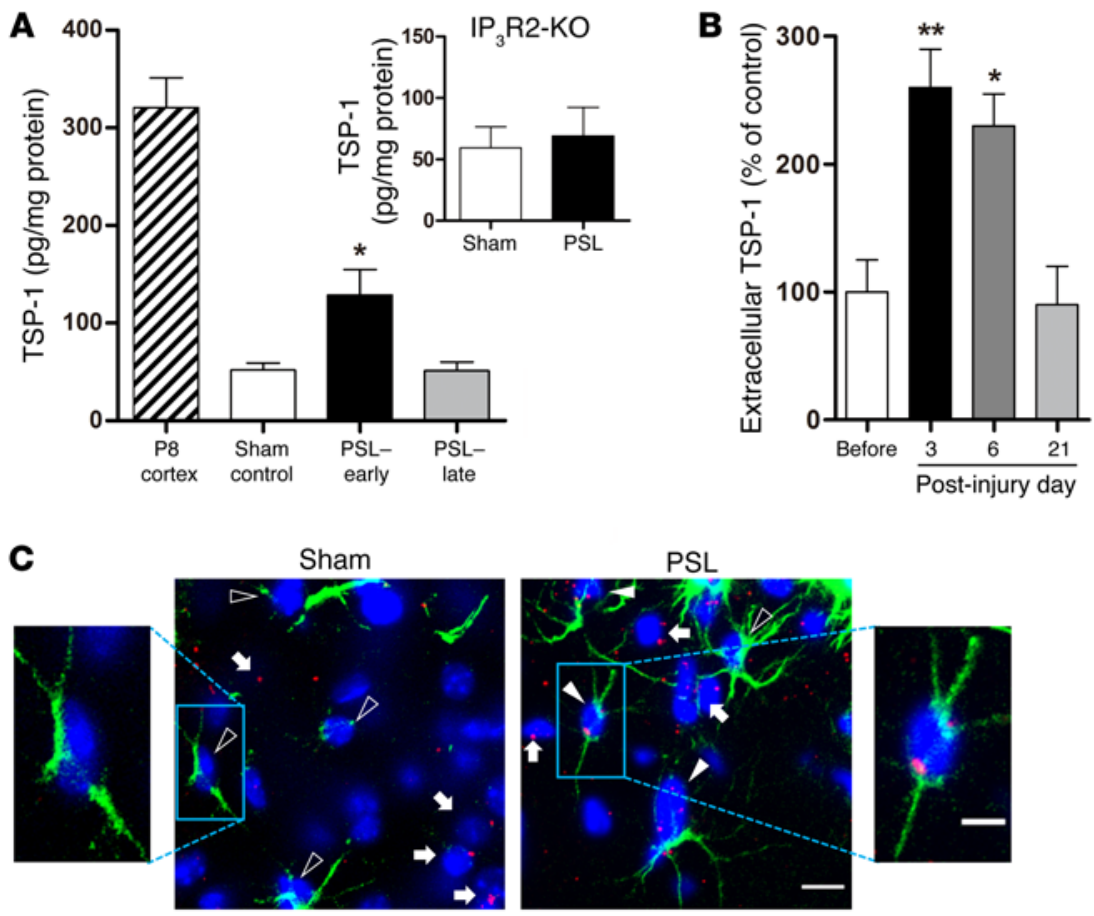

Tsp1/GFAP/DAPI
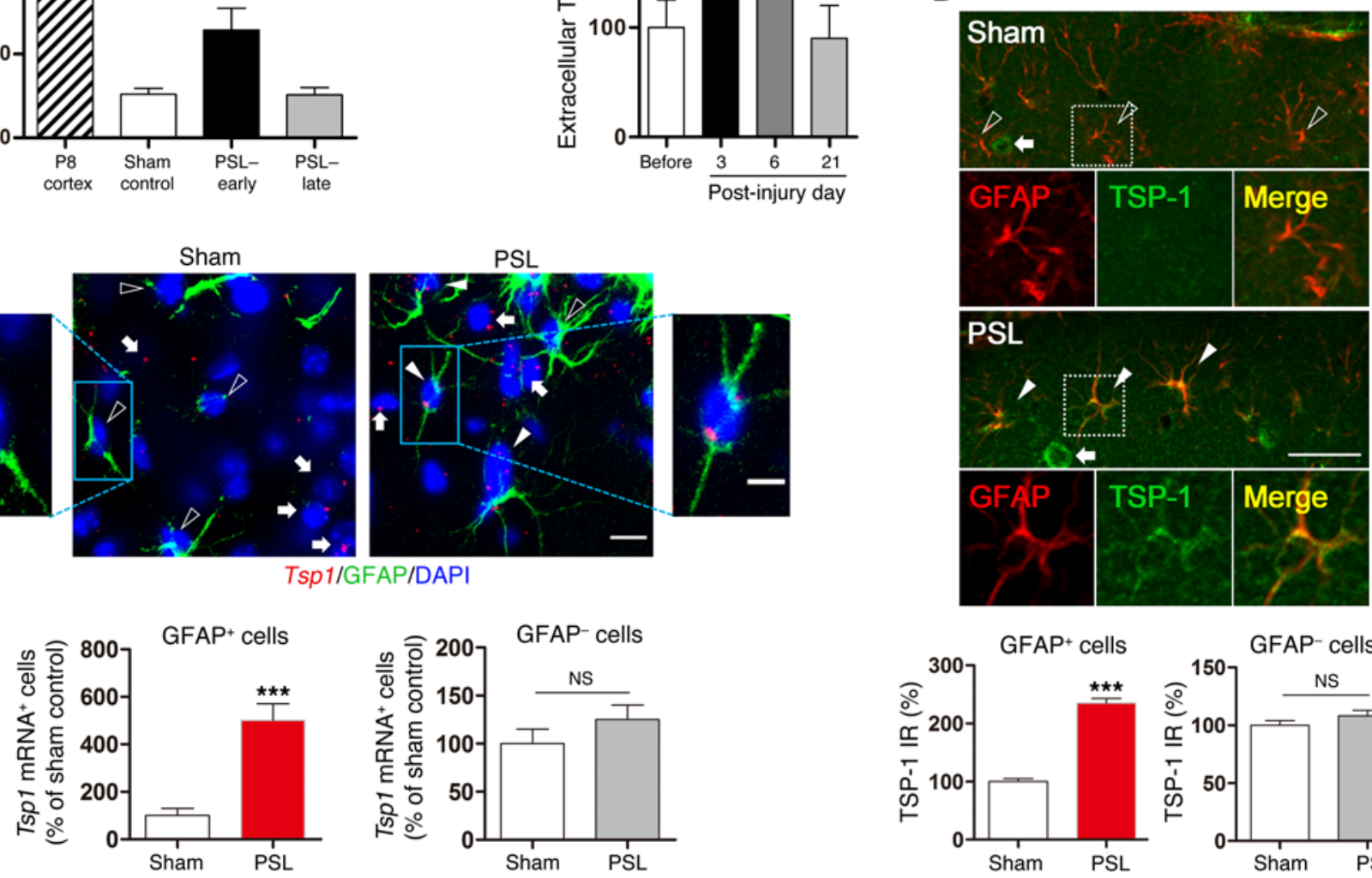

Figure 4. Astrocytic $\mathrm{Ca}^{2+}$-dependent upregulation of TSP-1 in S1 cortex following PSL injury. (A) TSP-1 protein levels in S1 cortex measured with ELISA in $\mathrm{P} 8$ positive control, sham control, PSL-early, and PSL-late mice. $n=3-4$ mice/group. ${ }^{*} P<0.05$ versus sham control or PSL-late mice, by 1-way ANOVA. Inset graph: S1 cortex TSP-1 levels in sham and PSL-early IP $\mathrm{R} 2-\mathrm{KO}$ mice $(n=3 /$ group). No significant difference was observed between groups $(P>0.05$, by unpaired $t$ test). (B) Extracellular TSP-1 levels in S1 cortex following PSL injury measured with ELISA using in vivo microdialysate samples. Extracellular TSP-1 levels significantly increased following PSL injury, peaked at 3 to 6 days after injury, and subsequently decreased to baseline levels ( $n=6$ mice/ group). ${ }^{*} P<0.05$ and ${ }^{*} P<0.01$ versus pre-injury levels, by 1-way ANOVA. (C) Top: Representative FISH images of Tsp1 mRNA (red) and immunohistochemical staining of astrocytes (GFAP, green) with DAPI staining (blue). Scale bars: $10 \mu \mathrm{m}$ and $5 \mu \mathrm{m}$ (magnified images). Solid arrowheads indicate representative GFAP' and Tsp1 mRNA ${ }^{+}$cells. Open arrowheads indicate representative GFAP' and Tsp1 mRNA $^{-}$cells. Arrows indicate representative GFAPand Tsp1 mRNA ${ }^{+}$cells. Bottom: Proportion of Tsp1 mRNA+ cells in GFAP+ and GFAP- cells normalized to the proportion in the sham group. $n=15$ image sections/5 mice for sham and 12 sections/4 mice for the PSL-injured group (3 sections/mouse). ${ }^{* * *} P<0.001$ and NS $(P>0.05)$, by Mann-Whitney $U$ test. (D) Immunofluorescence images of 51 cortex showing greater colocalization of astrocytes (GFAP, red) and TSP-1 (green) in PSL-injured mice (bottom) compared with sham controls (top). Scale bar: $20 \mu \mathrm{m}$. Dashed squares were expanded and separated (for GFAP, TSP-1, and merge) and are shown in the bottom images. Bottom graphs: Proportion of TSP-1 immunoreactivity (IR) colocalized with GFAP ${ }^{+}$astrocytes $\left(n=48\right.$ cells from 3 mice/group; ${ }^{* * *} P<0.001$, by unpaired $t$ test) or with GFAP- cells ( $n=75$ cells from 3 mice from the sham control group, 97 cells from 3 mice from the PSL-injured group; NS, $P>0.05$, by unpaired $t$ test). Error bars represent the mean \pm SEM.

jured) mice significantly increased new spine formation 1 day after the injection and induced a mechanical hypersensitivity that was apparent 1 day after injection and sustained for at least 1 month (Figure 5D). This allodynic effect was significantly inhibited by coinjection of gabapentin $(500 \mathrm{mg} / \mathrm{kg}$, i.p.; TSP- 1 alone, $n=6$ vs. TSP-1 plus gabapentin, $n=3$ [percentage of maximum pain score]: $10.8 \% \pm 2.0 \%$ vs. $13.3 \% \pm 1.7 \%$ at 0 days, $P=0.451 ; 53.3 \% \pm 6.4 \%$ vs. $23.3 \% \pm 10.9 \%$ at 3 days, ${ }^{*} P=0.038 ; 60.0 \% \pm 5.9 \%$ vs. $40.0 \%$ $\pm 2.9 \%$ at 7 days, $P=0.058 ; 65.0 \% \pm 5.2 \%$ vs. $31.4 \% \pm 4.4 \%$ at 14 days, ${ }^{* *} P=0.004$, by unpaired $t$ test), while injection of heat-inactivated TSP-1 had no effect (Supplemental Figure $4 \mathrm{C}$ ), both results confirming the specificity of the TSP-1 effect. Hence, these data strongly suggest that $\mathrm{Ca}^{2+}$-dependent release of TSP-1 from S1 astrocytes following PSL injury induces spine plasticity and subsequent mechanical allodynia.

Increased mGluR5 signaling in mature S1 astrocytes following PSL injury causes $\mathrm{Ca}^{2+}$-dependent TSP-1 release and subsequent mechanical allodynia. Finally, we sought to identify the mechanism linking PSL injury to the enhanced S1 astrocytic $\mathrm{Ca}^{2+}$ transients that then induce TSP-1 release, spine plasticity, and mechanical allodynia. We hypothesized that the enhanced peripheral afferent activity and excitatory signaling in the ascending pathway after nerve 
A

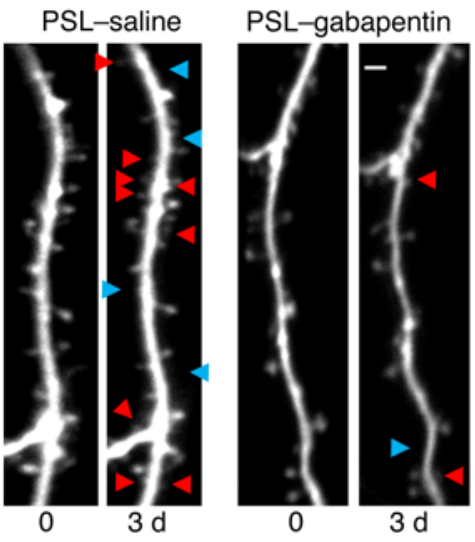

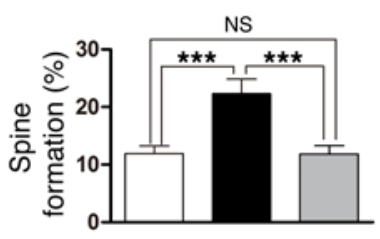

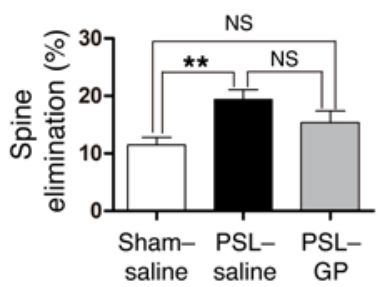

B

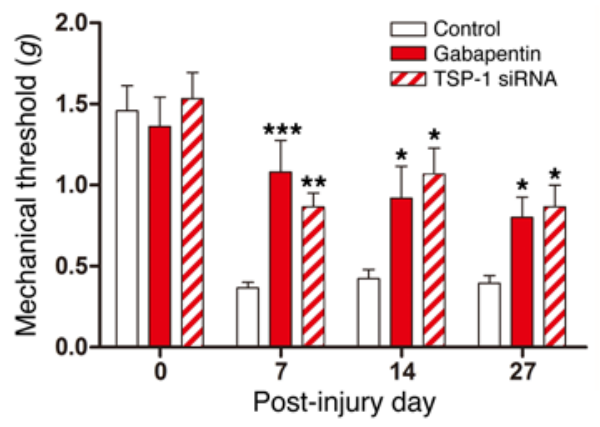

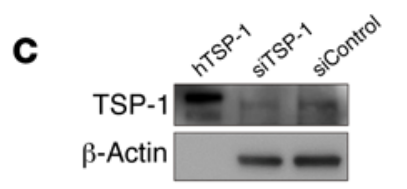

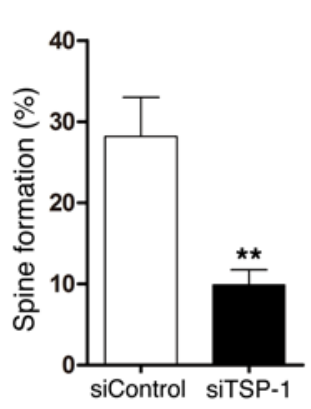

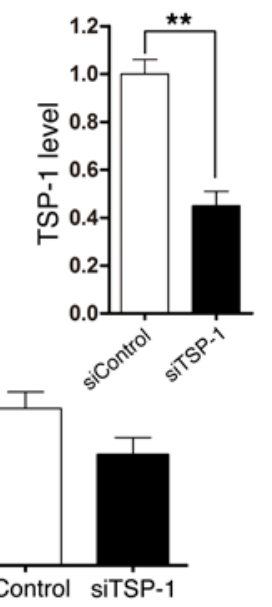
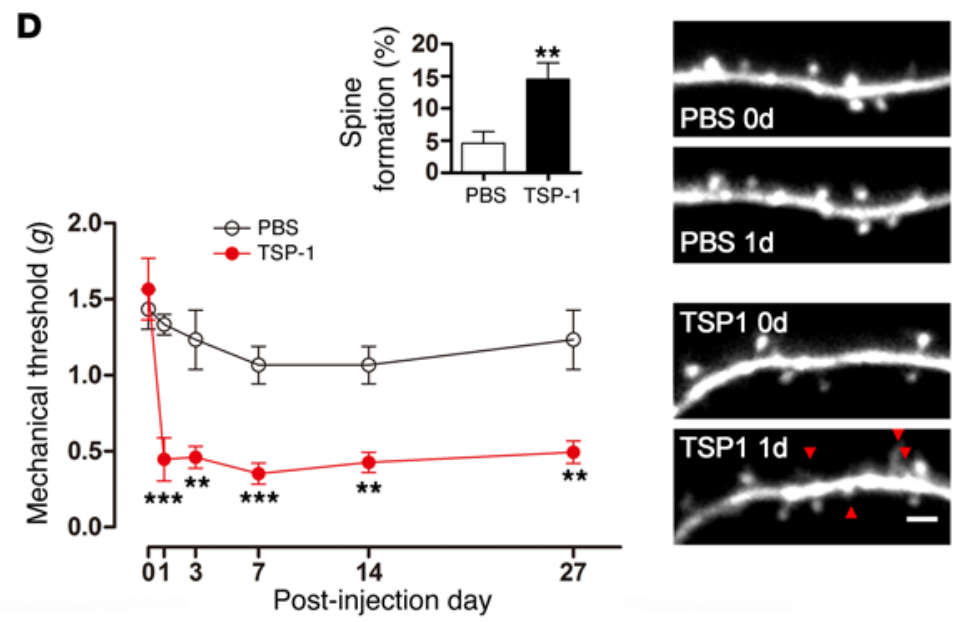

Figure 5. TSP-1 released from S1 astrocytes following PSL injury promotes synaptic rewiring and sustained mechanical allodynia. (A) Images of the same S1 apical dendrite before and 3 days after PSL injury with sustained (Elvax) saline or gabapentin application. Arrowheads indicate spine formation (red) and elimination (blue). Scale bar: $2 \mu \mathrm{m}$. Graphs: Spine formation (top) and elimination (bottom) rates in saline-administered, sham-operated mice ( $n=24$ dendrites/3 mice), PSL-injured mice ( $n=17$ dendrites $/ 3$ mice), and gabapentin-infused, PSL-injured mice $\left(n=21\right.$ dendrites $/ 3$ mice). ${ }^{* *} P<0.01$ and ${ }^{* *} P<0.001$, by 1 -way ANOVA. (B) Mean mechanical thresholds following PSL injury in control $(n=7)$, gabapentin-infused $(n=5)$, and TSP-1 siRNAinjected $(n=6)$ mice. ${ }^{*} P<0.05$, ${ }^{* *} P<0.01$, and ${ }^{* * *} P<0.001$ versus control, by 1 -way ANOVA. (C) Western blots demonstrate selective knockdown of TSP-1 expression in $\mathrm{S} 1$ cortex following TSP-1 siRNA injection. As for positive control of TSP-1, recombinant human TSP-1 (hTSP-1) was loaded (left band). Graphs: Quantitative analysis of Western blots $(n=4) .{ }^{* *} P<0.01$, by unpaired $t$ test. Spine formation (left) and elimination (right) rates in control siRNA-injected mice (siControl, $n=11$ dendrites/3 mice) and TSP-1 siRNA-injected mice (siTSP-1, $n=8$ dendrites $/ 2$ mice). ${ }^{* *} P<0.01$, by unpaired $t$ test. (D) Single injection of TSP-1 protein ( $n=6$ mice), but not PBS $(n=6)$, into S1 cortex induced mechanical hypersensitivity that lasted at least 4 weeks. ${ }^{* *} P<0.01$ and ${ }^{* * *} P<0.001$, by unpaired $t$ test. Images of the same S1 apical dendrite in naive mice before and 1 day after an injection of PBS (upper panels) or TSP-1 (lower panels). Arrowheads indicate spine formation. Scale bar: $2 \mu \mathrm{m}$. Inset graph: Spine formation rates in PBS-injected ( $n=8$ dendrites/3 mice) and TSP-1-injected ( $n=9$ dendrites $/ 3$ mice) mice. ${ }^{* *} P<0.01$, by unpaired $t$ test. Error bars represent the mean \pm SEM.

injury $(10,37,38)$ may be precipitating factors. In vivo microdialysis showed that extracellular glutamate levels in the S1 cortex were significantly increased following PSL injury, peaking 3 days after injury before subsequently returning to baseline levels (Figure 6A). We therefore investigated whether astrocytic glutamate receptors may be responsible for the $\mathrm{Ca}^{2+}$-dependent release of TSP-1. Initially, we used cultures of cortical astrocytes and found that application of glutamate $(100 \mu \mathrm{M})$ significantly increased TSP-1 protein expression (Figure 6B) and its release into the extracellular space (Figure 6C). BAPTA-AM blocked this increased TSP-1 expression (Figure $6 \mathrm{~B}$ ), indicating the dependence of TSP-1 expression on intracellular $\mathrm{Ca}^{2+}$. The glutamate-induced increase in TSP-1 expression was also blocked by mGluR antagonists and specifically by $\alpha$-methyl-4carboxyphenylglycine (MCPG), the nonselective group I/II mGluR antagonist, and by 2-methyl-6-(phenylethynyl)-pyridine (MPEP), the mGluR5 antagonist (Figure 6B). In contrast, 6-cyano-7-nitroquinoxaline-2,3-dione (CNQX, an $\alpha$-amino-3-hydroxy-5-methyl4-isoxazolepropionic acid [AMPA] receptor antagonist) or MK-801 (an NMDA receptor antagonist) had no effect (Figure 6B). MPEP also abolished the glutamate-evoked release of TSP-1 (Figure 6C). Local application of MPEP also blocked the mGluR5-mediated increase in TSP-1 expression in the S1 cortex in vivo following PSL injury (Figure 6D). Immunoelectron microscopic imaging showed that mGluR5 expression in glia was upregulated following PSL injury (Figure 6E).

A recent study (39) reported that mGluR5-mediated astrocyte somatic $\mathrm{Ca}^{2+}$ responses to glutamatergic transmission and mGluR5 expression levels were both downregulated during mouse 
A

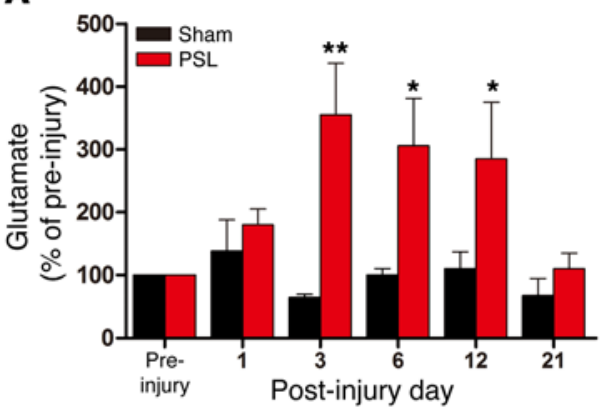

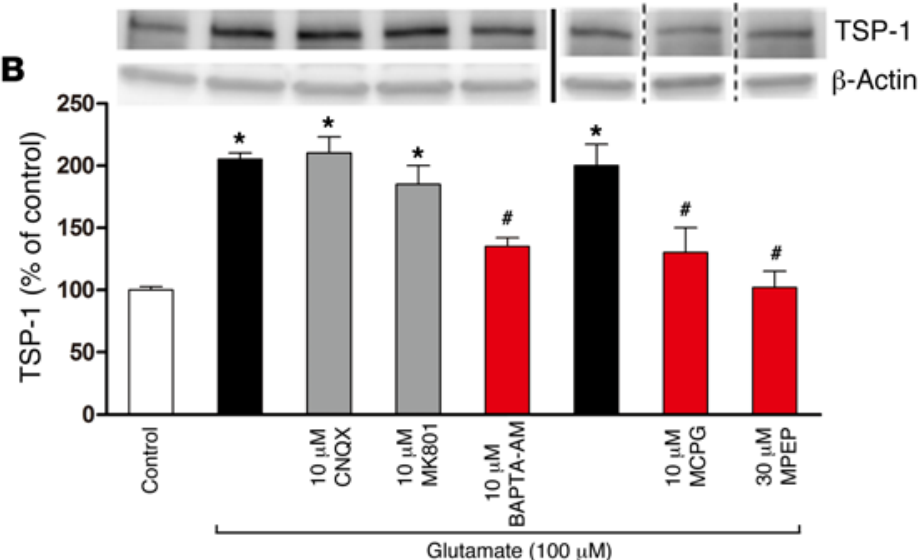

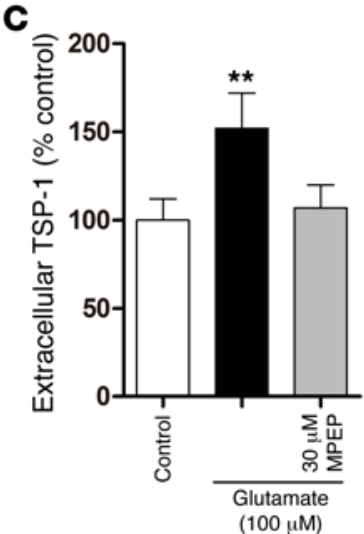

D

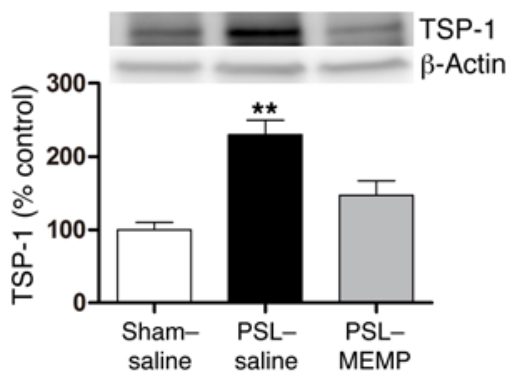

E

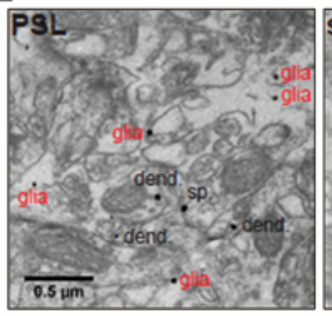

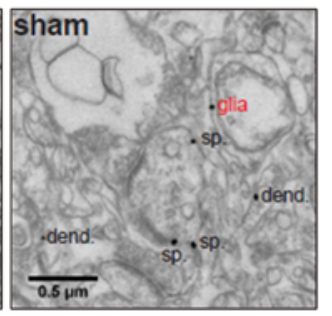

Figure 6. Increased extracellular glutamate and astrocytic mGluR5 signaling in S1 cortex following PSL injury mediates Ca ${ }^{2+}$-dependent TSP-1 release. (A) Extracellular glutamate levels in S1 cortex transiently increased after PSL injury ( $n=5$ mice/group), but not after sham operation ( $n=3$ mice). ${ }^{*} P<0.05$ and ${ }^{* *} P<0.01$ versus pre-injury, by 1-way ANOVA. (B) $100 \mu \mathrm{M}$ glutamate-mediated increases in TSP-1 expression in cultured cortical astrocytes, as measured with Western blots, were inhibited by the mGluR5 antagonists MCPG and MPEP and by BAPTA-AM, but were unaffected by AMPA and NMDA antagonists (CNQX and MK-801). ${ }^{*} P<0.05$ versus control; ${ }^{*} P<0.05$ versus glutamate, by Kruskal-Wallis test. Note that the MCPG and MPEP data were run on separate gels, as indicated by the vertical lines. (C) Levels of extracellular TSP-1 released from cultured cortical astrocytes as measured by ELISA. Glutamate application significantly increased TSP-1 release, which was blocked by MPEP. ${ }^{* *} P<0.01$, by Kruskal-Wallis test. (D) Local MPEP infusion in vivo blocked the increase in TSP-1 levels in the S1 cortex following PSL injury. Representative Western blots demonstrated a significant increase in S1 cortex TSP-1 levels following PSL injury, which was inhibited by MPEP administration into S1 cortex ( $n=4$ mice/group). ${ }^{* *} P<0.01$, by 1-way ANOVA. Error bars in A-D represent the mean \pm SEM. (E) Representative electron micrographs of contralateral (left) S1 cortex (layer I) labeled for mGluR5 in PSL-injured and sham control mice. Immunogold labeling for mGluR5 was found both in neuronal compartments (dend., dendrite; sp., spine) and glia, with the frequency of glia labeling increasing after PSL injury.

development, with only very small levels found in the cortex of adult mice. In contrast, the somatic $\mathrm{Ca}^{2+}$ responses of astrocytes to ATP remained intact during development (with a tendency to increase in the adult cortex as compared with that observed in the cortex of pups). Consistently, mature astrocytes in S1 cortical slices from sham-operated control mice showed only rare somatic $\mathrm{Ca}^{2+}$ responses to bath application of (S)-3,5-dihydroxyphenylglycine (DHPG, a group I mGluR agonist) (Figure 7A). In contrast, in slices isolated from PSL-injured mice (3-6 days after injury), the proportion of astrocytes responding to DHPG was significantly increased (Figure 7A). The astrocytic response to ATP was similar in sham control and PSL-injured mice (with a tendency to decrease in PSL-injured mice). Similarly, the proportion of astrocytes that showed somatic $\mathrm{Ca}^{2+}$ transients in response to topical application of DHPG to S1 cortex in vivo was markedly increased after PSL injury (Figure 7B). These results indicate an upregulation of S1 astrocytic mGluR5 signaling in the first week following peripheral nerve injury. Blocking mGluR5 signaling in the S1 cortex by Elvax-mediated local MPEP application significantly suppressed mechanical allodynia for at least 1 month (Figure 7C). The antiallodynic effect of MPEP application was evident 2 days after PSL injury (Supplemental Figure 5A). The PSL-induced spine turnover increase was also prevented by MPEP application (spine turnover rate: PSL-MPEP, $11.11 \% \pm 1.08 \%$ vs. PSL-saline, $20.85 \%$ $\pm 1.57 \%, n=16-17$ dendrites $/ 3$ mice/group, $P<0.001$, by unpaired $t$ test). These findings suggest that upregulated mGluR5 signaling in S1 astrocytes following peripheral nerve injury induces somatic $\mathrm{Ca}^{2+}$ transients and TSP-1 release, events that ultimately result in sustained mechanical allodynia (Supplemental Figure 6).

\section{Discussion}

Peripheral nerve injury triggers neuroplastic changes along the somatosensory nervous system, from the peripheral sensory afferents to the spinal cord, thalamus, and cortex, leading to chronic neuropathic pain, a debilitating disease with poor therapeutic prognosis (37). The role of neural circuits in the cortex in this 
A

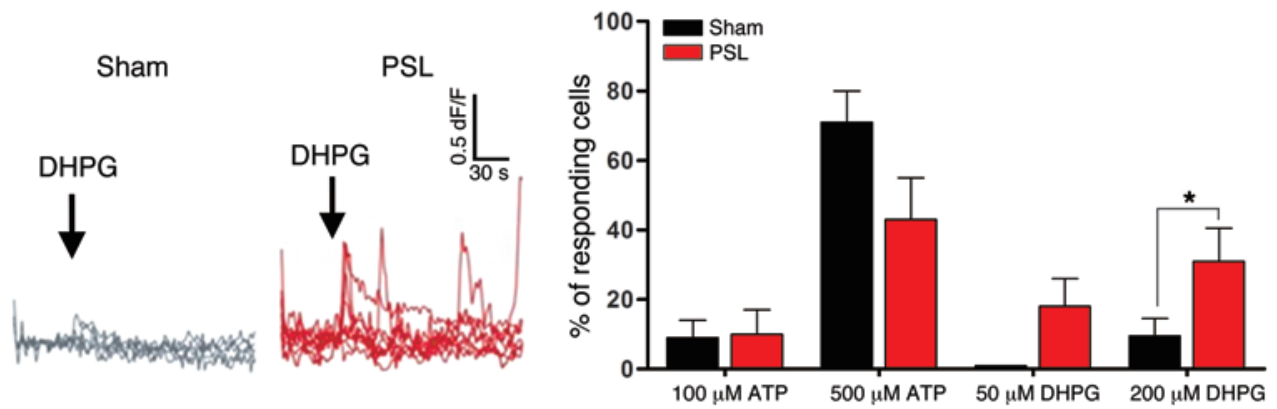

B

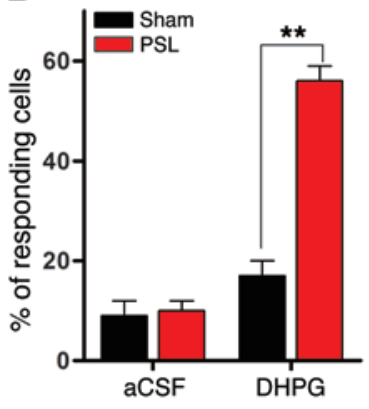

S1 astrocytes

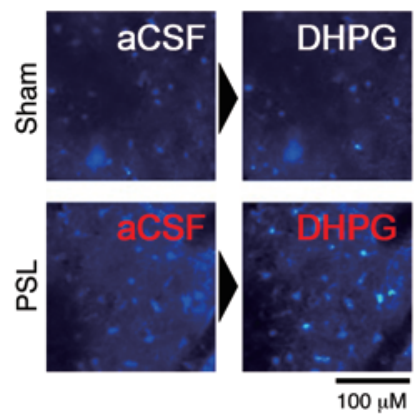

C

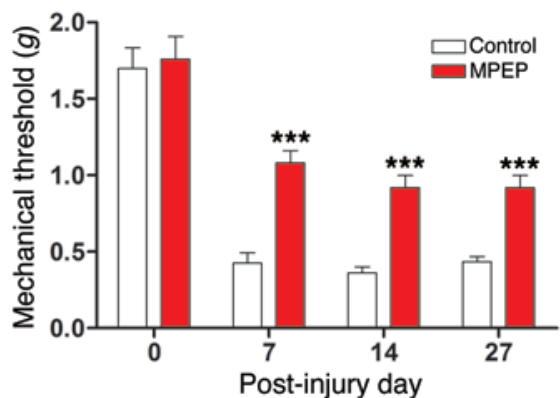

Figure 7. Reawakened mGluR5 signaling in S1 astrocytes following PSL mediates Ca ${ }^{2+}$ activation and long-lasting mechanical allodynia. (A) Proportion of $\mathrm{S} 1$ astrocytes in acute brain slices showing $\mathrm{Ca}^{2+}$ transients (left panel: representative $\mathrm{Ca}^{2+}$ traces) in response to puff application of ATP (100 and 500 $\mu \mathrm{M}$ ) or DHPG (group 1 mGluR agonist, 50 and $200 \mu \mathrm{M}$ ). ${ }^{*} P<0.05$, by Mann-Whitney $U$ test. $n=6$ (ATP, sham), 3 (ATP, PSL), 6 (DHPG, sham), or 7 (DHPG, PSL) brain sections from 2 to 4 mice/group. (B) Proportion of S1 astrocytes in vivo showing Ca ${ }^{2+}$ responses to local aCSF or DHPG application. $n=83$ cells/3 mice (sham) or 132 cells $/ 4$ (PSL-injured) mice. ${ }^{* *} P<0.01$, by Scheffe's $F$ test. Representative in vivo images are shown. (C) Mean mechanical thresholds following PSL injury in MPEP- $(n=6)$ or saline-treated $(n=5)$ mice. ${ }^{* *} P<0.001$, by unpaired $t$ test. Error bars represent the mean \pm SEM.

chronic pain behavior has been regarded as a passive reflection of the events occurring in the spinal cord (1). However, whole-brain imaging studies in patients with chronic neuropathic pain clearly identifies a range of plastic changes at the supraspinal level that include alterations of somatotopic maps, cortical thickness, and excitability in the S1 cortex $(15,40-42)$. Such anatomical and functional changes in the connectivity between the "pain matrix" cortical regions are reliable predictors for the severity of peripheral neuropathic pain $(43,44)$. Our results extend this to show that changes in cortical neural circuits are not simply passive reflections of ongoing altered afferent input from the spinal areas, but actively contribute to neuropathic mechanical allodynia. By revealing some of the underlying mechanisms, our study suggests that cortical changes may move beyond their utility as just diagnostic tools and serve as potential targets for therapeutics.

An important role of glial activation and the subsequent modification of neuronal activity in the pathogenesis of neuropathic pain have been extensively reported for the spinal cord $(45,46)$. In contrast, few studies have investigated the potential role of cortical glia-neuron crosstalk in chronic pain, perhaps partly due to the difficulty in revealing causality and mechanisms at a site so distant from the initial insult in the periphery. Previous histological studies, to our knowledge, have failed to show any distinct activation of glia (i.e., microglia and astrocytes) in the S1 cortex following peripheral nerve injury. Using in vivo 2-photon $\mathrm{Ca}^{2+}$ imaging in live animals, we demonstrate here that $\mathrm{S} 1$ cortical astrocytes are acutely and functionally activated following nerve injury. Further- more, the consistency of multiple approaches to blocking signaling from these activated S1 astrocytes to attenuate neuropathic mechanical allodynia and the induction of spine formation and mechanical allodynia by activation of astrocytic signaling in the absence of injury together reveal that the astrocyte-dependent new synaptic connections in S1 cortex substantially mediate sustained mechanical allodynia. This mechanism is probably specific to mechanical allodynia, since blockade of S1 astrocyte-induced synaptic plasticity had more minimal effects on thermal allodynia (data not shown).

Our findings also recall the structural synaptic plasticity implicated in long-term memory formation $(4,47)$. While changes in the efficacy of existing synapses contribute to short-term memory, new synaptic connections in the cortex are required for persistent memory storage $(3,4)$. We and others have previously focused on amplified excitatory transmission in the $\mathrm{S} 1$ cortex and anterior cingulate cortex following peripheral injury, the suppression of which temporarily relieves mechanical allodynia $(9,48)$. While chronic pain may include components of increased efficacy of preexisting connections, the structural synaptic plasticity we report here may also play a key role in mediating sustained mechanical hypersensitivity in neuropathic pain.

Close interactions between neurons and glia play a key role in information processing and diseases in the CNS $(23,45,46,49)$. Astrocytes form a structural network surrounding neurons and synapses and functionally maintain the homeostasis of ions and transmitters (23). They are not electrically excitable, but have a 
range of important signaling pathways including group I mGluRmediated somatic $\mathrm{Ca}^{2+}$ oscillations and TSP-1/2 release capacity during development, when neural circuits are forming. These signaling pathways are typically downregulated in the adult brain $(23,39)$. Here, we show that peripheral nerve damage results in a re-emergence of mGluR5 signaling in S1 cortical astrocytes and suggest that this re-emergent mGluR5 pathway defines a critical period of the $\mathrm{S} 1$ circuit plasticity that is associated with enhanced astrocytic $\mathrm{Ca}^{2+}$ activity and TSP-1 release and that enables neuropathic mechanical allodynia to become entrenched. Thus, we provide the evidence that cortical astrocytes are converted to an active, immature phenotype despite a lack of direct injury in the mature brain. This reawakened immature signaling in astrocytes reflects an abnormal pathological state of the S1 cortex in nerve-injured mice, as a number of other aspects of neuronal signaling revert back to the immature phenotype upon neuronal injury or stress. Hence, we propose that peripheral neuropathic pain represents, at least in part, an outcome of brain pathology (1). In this regard, it should be noted that an intrathecal injection of TSP-4 induced a reversible acute mechanical hypersensitivity ( 1 week's duration) through spinal neuron sensitization (50), whereas an injection of TSP-1 into S1 cortex in our current study resulted in long-lasting mechanical allodynia ( $>1$ month's duration). This suggests that cortical plasticity can cause more sustained enhanced nociceptive signaling. However, our results do not refute a role for the well-documented mechanisms outside of the S1 cortex (e.g., peripheral/spinal sensitization) that contributing to allodynia. There might be development of some cortexdependent allodynia beginning approximately 2 days after nerve injury, with earlier allodynia (day 1) likely due to spinal cord and/ or peripheral changes (Supplemental Figure 5A). Just how peripheral and spinal changes may combine or interact with cortical plasticity in the development and maintenance of chronic allodynia will be important to resolve.

It should, however, be recognized that interactions between neurons and astrocytes are bidirectional. Astrocytes respond to sensory inputs or synaptic activity with intracellular $\mathrm{Ca}^{2+}$ rises and remodeling of perisynaptic astrocytic processes, resulting in the release of neuroactive substances that may not only acutely affect synaptic and neuronal activity, but may also result in slower and more chronic aspects of neuronal plasticity (23, 51-55). Indeed, slower astrocyte-neuron interaction is more consistent with the temporal dynamics of astrocytic $\mathrm{Ca}^{2+}$ waves, which are much slower than the rapid time course of synaptic transmission (53). Astrocytes secrete a number of molecules that are not defined as gliotransmitters but that nevertheless directly modulate synaptic structures and thereby synaptic function (23) on a slower time scale. We focused on one class of these, the TSPs, because (a) they bind to synaptic $\alpha_{2} \delta$-1 receptors to induce excitatory synaptogenesis in vitro and in vivo; (b) gabapentin, which inhibits this synaptogenic action by antagonizing TSP binding to $\alpha_{2} \delta-1$, is effective against neuropathic pain (32); (c) dendritic spine malformations and reduced synaptic density in Down syndrome brain are directly linked to deficits in astrocytic TSP-1 protein expressions (56); and (d) astrocytes near the injured neuronal cell bodies release TSPs to cause functional and structural synaptic plasticity in vitro and in vivo $(50,55)$. We found that TSP-1 release from S1 astrocytes is markedly increased in the early post-injury phase, which is dependent on mGluR5mediated astrocytic $\mathrm{Ca}^{2+}$ signaling, before subsequently declining during the late post-injury phase. Inhibition of TSP-1 transcription or binding to neuronal $\alpha_{2} \delta-1$ by siRNA or gabapentin, respectively, substantially suppressed new spine formation and mechanical allodynia following PSL injury. These results indicate that TSP-1 is a molecular factor that causally links enhanced $\mathrm{S} 1$ astrocytic $\mathrm{Ca}^{2+}$, synaptic rewiring, and neuropathic pain.

In summary, our findings identify a sequence of events in the somatosensory cortex, a remote region not directly affected by the injured periphery and spinal cord, that contribute to mechanical hypersensitivity in neuropathic pain. Our proposed mechanistic model is summarized schematically in Supplemental Figure 6. Peripheral nerve injury results in afferent nerve hyperactivity (10, $37,38)$ that increases extracellular glutamate levels in the S1 cortex to activate mGluR5s and initiate cell signaling that results in astrocytic $\mathrm{Ca}^{2+}$ transients. Elevation of astrocytic $\mathrm{Ca}^{2+}$ levels then causes the release of TSP- 1 , which activates the neuronal $\alpha_{2} \delta$-1 receptor to induce new synaptic connections, possibly connecting innocuous S1 neural circuits to noxious circuits. Such maladaptive synaptic rewiring may contribute to the enhanced response of S1 circuits to intracortical stimuli and to innocuous tactile stimuli $(9,10)$, resulting in sustained mechanical allodynia. Blocking either nerve injury-induced early $\mathrm{S} 1$ astrocytic $\mathrm{Ca}^{2+}$ activation (e.g., by MPEP, $\mathrm{IP}_{3} \mathrm{R} 2-\mathrm{KO}, \mathrm{BAPTA}-\mathrm{AM}$, or fluoroacetate) or subsequent neuronal synaptic rewiring (e.g., by TSP-1 siRNA or gabapentin) prevents the full development of mechanical allodynia. Thus, appreciation of these astrocyte-mediated changes in cortical synaptic connections requires a paradigm shift in our understanding of neuropathic pain pathophysiology, one that may result in novel therapeutic strategies to treat debilitating neuropathic mechanical allodynia.

\section{Methods}

All mice were housed in cages (3-4 mice per cage) with ad libitum access to food and water. After the cranial window implantation, however, the mice were single caged to avoid any possible damage to the glass window. The room was maintained on a 12-hour light/12-hour dark cycle.

PSL injury and behavioral testing. Under isoflurane anesthesia (2.0\%), the right sciatic nerve of 3-month-old male C57BL/6 mice was exposed at the upper-thigh level and one-third to one-half of the diameter of the nerve was ligated with a 9-0 suture (57). In sham-operated mice, the nerve was exposed but left intact. For assessment of tactile allodynia, the mice were habituated for 30 minutes in a transparent plastic box with a wire mesh floor before pressing a set of 8 calibrated von Frey hairs (ranging from $0.07 \times g$ to $4.0 \times g$ of bending force) perpendicularly against the plantar surface of the hind paw until slight buckling was observed. The $50 \%$ paw withdrawal threshold was determined by the up-down method (58). $\mathrm{IP}_{3} \mathrm{R} 2-\mathrm{KO}$ mice (Itpr2 $2^{-/}$) and their littermate WT mice $\left(^{+/+}\right)$showed no different baseline mechanical sensitivity as compared with normal C57BL/6 mice. Thus, the behavioral data from both the $\mathrm{IP}_{3} \mathrm{R} 2^{+/+}$littermates and C57BL/ 6 control mice were pooled to form a control group (Figure 1F). The experimenters were blinded to genotyping and nerve injury. In a different set of experiments, we measured the hind-paw withdrawal response sensitivity (percentage of maximum pain score) as described previously (59). Briefly, the mice were brought into the behavior room 1 hour before the test session to allow them to habituate to the environment. 
To quantify the sensitivity to a tactile stimulus, von Frey filaments were applied to the plantar surface of the hind paw, and the paw withdrawal response was evaluated by scoring as follows: 0 , no response; 1 , a withdrawal response away from the stimulus with slight flinching and/or licking; and 2, an intense withdrawal response away from the stimulus with brisk flinching and/or licking. One trial involved 10 filament applications separated by 3 to 4 seconds, and the response to each application was scored as 0,1 , or 2 . The total score for each trial was summed, giving a range of scores of 0 to 20. The time course of mechanical allodynia following PSL injury as measured using both methods was almost the same (Figure 1C and Supplemental Figure $4 \mathrm{C}$ ). The behavioral tests were performed during the daytime.

Open-skull chronic cranial window implantation and long-term in vivo 2-photon imaging of spine dynamics. To implant the cranial window for chronic imaging (60), 2-month-old male C57BL/6 mice expressing EGFP under the control of the Thy1 promoter in a small subset of cortical neurons (GFP-M line mice) (31) were anesthetized with an i.p. injection of ketamine $(0.13 \mathrm{mg} / \mathrm{g})$ and xylazine $(0.01 \mathrm{mg} / \mathrm{g})$, and body temperature was maintained at $37^{\circ} \mathrm{C}$ using a heating pad. Dexamethasone $(0.04 \mathrm{ml}$ at $2 \mathrm{mg} / \mathrm{ml})$ was administered s.c. prior to surgery to prevent cerebral edema. Following scalp incision and cleaning, a circular craniotomy (2-3 $\mathrm{mm}$ in diameter) was performed on the skull above the hind-limb area of the left $\mathrm{S} 1$ cortex $(0.5 \mathrm{~mm}$ posterior and $1.5 \mathrm{~mm}$ lateral from the bregma) and covered with a glass coverslip.

In vivo imaging experiments (at 3-day intervals) began 3-4 weeks after the cranial window implantation. Isoflurane anesthesia (1.5\%) was used during each imaging session, which typically lasted 30 to 40 minutes. In the first imaging session, the position of the S1 hindpaw area was functionally determined using intrinsic optical signal imaging as described previously (10). A Ti:sapphire laser (Mai Tai HP; Spectra-Physics) was tuned to the excitation wavelength for GFP (950 $\mathrm{nm})$. Low-magnification image stacks $(512 \times 512$ pixels, $0.41 \mu \mathrm{m} /$ pixel, 250-400 optical planes, 2 - to $-2.5-\mu \mathrm{m} \mathrm{z} \mathrm{step)} \mathrm{of} \mathrm{fluorescently} \mathrm{labeled}$ cells inside the identified $\$ 1$ region were collected on the second imaging session using a FV1000MPE laser scanning microscope and a water-immersion objective lens ( $\times 60$; NA 0.9; Olympus) and matched to the blood vessel pattern on the brain surface to enable subsequent relocation of the imaged region. High-magnification, long-term timelapse imaging $(512 \times 512$ pixels, $0.08 \mu \mathrm{m} /$ pixel, $15-30$ optical planes, $0.5-\mu \mathrm{m} z$ step) of spine dynamics began on the third session and continued for 2 to 3 weeks.

MetaMorph (Molecular Devices) and ImageJ software (NIH) were used to analyze individual spines on the same dendritic segments from 3D image stacks. Analysis was performed by an experimenter was blinded to the experimental conditions. As described previously (10), all types of dendritic protrusions were included in the analysis, except for the spines on dendritic branch tips. The rates of spine formation and elimination were determined as the percentages of spine gain and loss, respectively, between 2 successive imaging sessions, relative to the total number of spines in the former session. Spine turnover was defined as the total sum of gained and lost spines over a 3-day period, divided by twice the total number of initial spines. The spine turnover change in the S1 hind-paw area following PSL injury was region specific, because little change was found in the barrel somatosensory cortex (Supplemental Figure 5B).

Dye loading and in vivo 2-photon $\mathrm{Ca}^{2+}$ imaging. Dye loading and $\mathrm{Ca}^{2+}$ imaging of $\mathrm{S} 1$ astrocytes were performed through a small open- skull cranial window under continuous visual guidance with a 2-photon microscope, with modification of the Stosiek et al. protocol (61). Briefly, a total of 0.8 mM Oregon Green 488 BAPTA-1-AM (OGB-1AM) was dissolved in DMSO with 20\% pluronic acid (Invitrogen) and mixed in artificial CSF containing SR101 (Sigma-Aldrich). A standard patch-clamp pipette was filled with this solution and inserted into S1 cortex to a depth of 10 to $100 \mu \mathrm{m}$ from the pial surface during urethane anesthesia $(1.9 \mathrm{~g} / \mathrm{kg}$, i.p.) with atropine $(0.4 \mathrm{mg} / \mathrm{kg}$, i.p.). The dye solution was then pressure ejected (5-10 psi; IM-300; Narishige) from the pipette for 1 minute. After loading, the pipette was withdrawn and the cranial window sealed with a glass coverslip. $\mathrm{Ca}^{2+}$ fluorescence in S1 astrocytes and/or neurons was imaged using the FV1000MPE (Olympus), A1R MP (Nikon), or LSM $7 \mathrm{MP}$ (Zeiss) 2-photon microscope with the Mai Tai DeepSee (Spectra-Physics) Ti:sapphire laser (power: under $10 \mathrm{~mW}$ ) at an excitation wavelength of 800 to $850 \mathrm{~nm}$. Excitation light was focused using a water-immersion objective ( $\times 60$, NA 0.9, Olympus; 25×, NA1.1, Nikon). Time-lapse images of S1 astrocytic $\mathrm{Ca}^{2+}$ fluorescence were recorded at $0.5 \mathrm{~Hz}$ for 10 minutes. The baseline fluorescence intensity of $\mathrm{F}_{0}$ was obtained by averaging the lower two-thirds of the fluorescence intensity values for each astrocyte. $\mathrm{A} \mathrm{Ca}^{2+}$ transient (event) was defined as a fluorescence intensity change above $15 \%$ of the baseline value. The amplitude of the $\mathrm{Ca}^{2+}$ fluorescence signal was calculated as $\Delta \mathrm{F} / \mathrm{F}_{0}\left(\Delta \mathrm{F}=\mathrm{F}-\mathrm{F}_{0}\right)$.

Preparation and implantation of Elvax drug solutions. As described previously (62), beads (100 mg) of Elvax (DuPont) were dissolved in dichloromethane $(100 \mathrm{mg} / \mathrm{ml})$ and mixed with $10 \mu \mathrm{l}$ DMSO containing $2 \%$ fast green and $10 \mu \mathrm{l}$ solution containing either $20 \mathrm{mM}$ BAPTA-AM, $100 \mathrm{mM}$ fluoroacetate, $50 \mathrm{mM}$ gabapentin, $5 \mathrm{mM}$ MPEP, $1 \mathrm{mM}$ SR101, or $0.9 \%$ normal saline. After stirring until homogenous, the Elvax solution was plated on a glass dish, frozen quickly, kept at $-70^{\circ} \mathrm{C}$ for 1 hour, and then placed at $-20^{\circ} \mathrm{C}$ overnight to allow the dichloromethane to evaporate. Final concentrations of the drugs in Elvax were approximately $2 \mathrm{mM}$ (BAPTA-AM), $10 \mathrm{mM}$ (fluoroacetate), $5 \mathrm{mM}$ (gabapentin), $500 \mu \mathrm{M}$ (MPEP) and $100 \mu \mathrm{M}$ (SR101). A small piece of drug-soaked Elvax $(2 \mathrm{~mm} \times 2 \mathrm{~mm})$ was placed on the dura matter through an open-skull cranial window. The implantation of Elvax containing saline or the above drugs did not cause deficits in general motor behaviors. The estimated duration of drug delivery using Elvax is approximately 6 days (10).

Drug and siRNA delivery into $S 1$ cortex and repeated dendrite imaging. Under ketamine $(0.13 \mathrm{mg} / \mathrm{g})$ and xylzine $(0.01 \mathrm{mg} / \mathrm{g})$ anesthesia, an open cranial window was made over the left S1 hind-limb area of 3-month-old male M-line mice after scalp incision and lightly sealed with a glass coverslip using a $3 \mathrm{M}$ Vetbond. Several apical dendrites of layer $\mathrm{V}$ pyramidal neurons were then randomly selected by the experimenter, who was unaware of the subsequent experimental conditions, and imaged at high magnification $(512 \times 512$ pixels, $0.08 \mu \mathrm{m} / \mathrm{pixel}$, 15-30 optical planes, $0.5-\mu \mathrm{m} z$ step). Then, the coverslip was removed, and a piece of drug-soaked Elvax was placed on the dura. The margin of the cranial window that was not covered with the Elvax was sealed with Kwik-Sil (World Precision Instruments), and the incised scalp was sutured. Three days after the first imaging session, the scalp was incised again and the Elvax and Kwik-Sil removed. The cranial window was then sealed with a coverslip, and high-magnification imaging of the same dendrites was performed (Figure 3A). For repeated dendrite imaging before and 3 days after siRNA injection, the same protocol was used, except that instead of Elvax implantation, approximately 
$0.5 \mu \mathrm{l}$ TSP-1 or control siRNA was injected into S1 cortex using a glass patch-clamp micropipette. Mouse TSP-1 siRNA (catalog sc-36666; Santa Cruz Biotechnology Inc.) and control siRNA (catalog sc-36869; Santa Cruz Biotechnology Inc.) were prepared in a $20-\mu \mathrm{M}$ solution and injected using the siRNA delivery reagent jetSI (10 mM; Polyplus Transfection) according to the manufacturer's in vivo transfection protocol described previously (63).

In vivo 2-photon $\mathrm{Ca}^{2+}$ uncaging and repeated dendrite imaging. For 2-photon photolysis of $\mathrm{Ca}^{2+}$ within an astrocyte soma and simultaneous repeated imaging of dendrites, the Twin Scanner FV1000MPE microscope (Olympus) with 2 independent 2-photon lasers (Spectra Physics) were used. M-line mice were anesthetized with urethane (1.9 $\mathrm{g} / \mathrm{kg}$ ) and atropine $(0.4 \mathrm{mg} / \mathrm{kg}$, i.p.). An open craniotomy was performed over the left $\mathrm{S} 1$ hind-limb region, and OGB-1-AM was loaded by a bolus injection into layer I. The dura was then removed and caged $\mathrm{Ca}^{2+}$ (DMNP-EDTA, $\left.2 \mathrm{mM}\right)$ and SR101 $(100 \mu \mathrm{M})$ were topically infused over the cortical surface for 20 minutes. Two-thirds of the cranial window was sealed with a glass coverslip, and DMNP-EDTA was topically applied through the remaining exposed region every 30 minutes. Photolysis of caged $\mathrm{Ca}^{2+}$ was done at 5-minute intervals using $730 \mathrm{~nm}$ 2-photon laser stimulation (10 mW, 50-ms duration; Tornado mode; Olympus) focused within an astrocyte soma (Figure 2). The same laser stimulation without DMNP-EDTA application did not induce astrocytic $\mathrm{Ca}^{2+}$ transients. Excessive $\mathrm{Ca}^{2+}$ uncaging with 1- or 2-minute stimulation intervals induced morphological changes of the stimulated astrocytes, and hence the more mild activation protocol was used. Two-photon (900 nm) time-lapse, high-magnification imaging of the same dendritic segments was performed at 30-minute intervals between the uncaging procedures (Figure 2). To avoid possible damage to dendritic structures by penetration of the glass pipette into the $\mathrm{S} 1$ cortex, the $\mathrm{Ca}^{2+}$ dye was bolus injected into a region remote from the area of imaging and uncaging. Prior to acquisition of experimental data, we confirmed the success of the uncaging protocol by photostimulating $(730 \mathrm{~nm})$ the center of an astrocyte soma, loaded with OGB-1-AM and SR101, and observing the simultaneous $\mathrm{Ca}^{2+}$ fluorescence response (900 nm, 0.5 Hz) (Figure 2A).

ELISA, IHC, and microdialysis. For ELISA, brain samples containing S1 layer I were micropunched and homogenized in ice-cold TBS with protease inhibitors. The homogenates were centrifuged for 10 minutes at $12,000 \times g$ and $4^{\circ} \mathrm{C}$, and the supernatants were transferred into EP tubes and stored at $-80^{\circ} \mathrm{C}$ until use. TSP levels were measured using the mouse ELISA kits (catalog SEA611Mu for TSP-1; catalog SED822Mu for TSP-2; and catalog SED824Mu for TSP-4; USCN Life Science) according to the manufacturer's protocol. Cortices from P8 mice that are known to express high TSP-1/2 levels (34) were used as a positive control.

For IHC, mice were perfused with $4 \%$ paraformaldehyde, and brains were excised and incubated with $4 \%$ paraformaldehyde for 24 hours before freezing in an embedding compound on dry ice. Frozen brains were cut with a cryostat (CM 1100; Leica) at $30 \mu \mathrm{m}$ thickness. Sections were incubated overnight at $4^{\circ} \mathrm{C}$ with the primary Abs mouse anti-TSP-1 (1:50; catalog MA5-13398; Thermo Fisher Scientific); rabbit anti-GFAP (1:1,000; catalog AB5804; EMD Millipore); rabbit antiIba1 (1:1,000; catalog 019-19741; Wako); rabbit anti-PSD-95 (1:500; catalog 51-6900; Invitrogen); and guinea pig anti-VGlut1 (1:2,500; AB230175; EMD Millipore), and then washed with PBS and further incubated for 3 hours at room temperature with the secondary Abs
Alexa Fluor 488-, Alexa Fluor 546-, and Alexa Fluor 594-conjugated mouse/guinea pig and rabbit IgGs (all from Invitrogen). Immunofluorescence images were obtained using a confocal microscope with FLUOVIEW (Olympus). The number of colocalized synaptic puncta (VGlut1/PSD-95) was quantified as previously described (24). Briefly, 5 - $\mu$ m-thick confocal Z-stacks (optical section depth of $0.33 \mu \mathrm{m} ; 15$ sections/Z-stack; imaged area measuring 11,272 $\mu \mathrm{m}^{2}$ ) of the synaptic zone in $\mathrm{S} 1$ cortex were imaged. Maximum projections of 3 consecutive optical sections were generated from the original Z-stack. The number of pre-, post-, and colocalized synaptic puncta was quantified by using the Puncta Analyzer plugin (developed by Barry Wark and provided by Cagla Eroglu) for Image J software. Three image stacks per mouse ( $n=4$ mice/group) were used for analyses.

For in vivo microdialysis, a probe (CXI-2-1; Eicom) was inserted into S1 cortex and perfused with artificial cerebrospinal fluid (aCSF) of the following composition: $125 \mathrm{mM} \mathrm{NaCl}, 5 \mathrm{mM} \mathrm{KCl}, 10 \mathrm{mM}$ glucose, $10 \mathrm{mM}$ HEPES, $2 \mathrm{mM} \mathrm{CaCl}_{2}$, and $2 \mathrm{mM} \mathrm{MgSO}_{4}$ (pH 7.4). Perfusion was performed continuously at a flow rate of $1.5 \mu \mathrm{l} /$ minute, with the perfusate collected for 105 minutes. The concentration of extracellular glutamate was measured by using the Amplex Red Glutamic Acid/Glutamate Oxidase Assay Kit (Invitrogen) according to the manufacturer's protocol.

Cell culture and Western blotting. Cortical astrocytes were prepared and cultured as described previously (64), with minor modifications. Cortical astrocytes were dissected from neonatal C57BL/6 mice, and cell digestion was done with $0.025 \%$ trypsin-EDTA. To purify astrocytes from cortical cultures, the cells were subjected to 24 hours of continuous shaking 10-14 days after plating to remove detached cells. For Western blotting, cells were seeded on 6-well cell culture plates at a density of $1 \times 10^{5}$ cells per well.

After glutamate stimulation with or without drugs, astrocytes were lysed, resolved with 10\% SDS-PAGE gels, and transferred to PVDF membranes. The membranes were blocked for 1 hour in Tris-buffered saline containing $0.1 \%$ Tween-20 (TBS/T) and 5\% BSA at room temperature. The membranes were then incubated overnight at $4^{\circ} \mathrm{C}$ with the primary Ab mouse anti-TSP-1 (1:50; Thermo Fisher Scientific), which was diluted with the immunoreaction enhancer solution Can Get Signal Solution 1 (TOYOBO). After TBS/T washes, the membranes were incubated for 1 hour at room temperature with HRP-conjugated anti-mouse Ab (1:20,000; Amersham Pharmacia Biotech), which was diluted with Can Get Signal solution 2 (TOYOBO). The membranes were washed with TBS/T, and the proteins were visualized by chemiluminescence.

$\mathrm{Ca}^{2+}$ imaging in brain slice. Coronal slices $(300 \mu \mathrm{m})$ that included the $\mathrm{S} 1$ cortex hind-limb area were cut from 5 -week-old male mice in a solution containing $87 \mathrm{mM} \mathrm{NaCl}, 25 \mathrm{mM} \mathrm{NaHCO}, 2.5 \mathrm{mM} \mathrm{KCl}$, $1.25 \mathrm{mM} \mathrm{NaH}_{2} \mathrm{PO}_{4}, 25 \mathrm{mM}$ D-glucose, $75 \mathrm{mM}$ sucrose, $7 \mathrm{mM} \mathrm{MgCl}$, and $0.5 \mathrm{mM} \mathrm{CaCl}_{2}$ saturated with $95 \% \mathrm{O}_{2}$ and $5 \% \mathrm{CO}_{2}$. Slices were incubated at approximately $34^{\circ} \mathrm{C}$ for 30 minutes and subsequently stored at room temperature in aCSF composed of $126 \mathrm{mM} \mathrm{NaCl}, 2.5$ $\mathrm{mM} \mathrm{KCl}, 1.3 \mathrm{mM} \mathrm{MgCl}_{2}, 10 \mathrm{mM}$ D-glucose, $2.4 \mathrm{mM} \mathrm{CaCl}_{2}, 1.24 \mathrm{mM}$ $\mathrm{NaH}_{2} \mathrm{PO}_{4}$, and $26 \mathrm{mM} \mathrm{NaHCO}_{3}$. Fluo-4 AM ( $3 \mu \mathrm{l}, 2 \mathrm{mM}$ in DMSO; Life Technologies) was dropped onto slices in a 1-ml aCSF bath containing 0.02\% Pluronic F-127 (Life Technologies). The slices were incubated with $6 \mu \mathrm{M}$ (final concentration) Fluo-4 AM for 60 minutes and then transferred to dye-free aCSF for 30 minutes. A Fluo-4-loaded slice was transferred to a submerged recording chamber superfused at a rate of 2 to $3 \mathrm{ml} /$ minute with aCSF. Cells were imaged using a micro- 
scope (BX51WI; Olympus) with a water-immersion objective $(x 40$, NA 0.8; Olympus), a CSU-10 laser scanner unit (Yokogawa), and an electron-multiplying charge-coupled device (EMCCD) camera (iXon; Andor) with excitation and emission wavelengths of 488 and $568 \mathrm{~nm}$, respectively. Astrocytes were selected from S1 layer I, typically 30-40 $\mu \mathrm{m}$ from the slice surface. Agonists were filled into micropipettes (tip diameter $\sim 2 \mu \mathrm{m}$ ) connected to a Pneumatic Picopump (World Precision Instruments) and delivered with a pressure pulse (4 psi, $500 \mathrm{~ms}$ ). Agonists were dissolved in HEPES solution containing $136 \mathrm{mM} \mathrm{NaCl}$, $2.5 \mathrm{mM} \mathrm{KCl}, 1.3 \mathrm{mM} \mathrm{MgCl}, 2.4 \mathrm{mM} \mathrm{CaCl}_{2}, 1.24 \mathrm{mM} \mathrm{NaH}_{2} \mathrm{PO}_{4}, 10$ $\mathrm{mM}$ HEPES, and $10 \mathrm{mM}$ glucose (pH 7.4). In some experiments, 100 $\mu \mathrm{M}$ Alexa Fluor 568 (Life Technologies) was applied to visualize the delivery. $\mathrm{Ca}^{2+}$ transients were measured by plotting the intensity of the regions of interest (ROI) over time, after a background ROI intensity had been subtracted. Data were analyzed using Origin 8 (OriginLab), ImageJ, and GraphPad InStat 3.10 (GraphPad Software).

Immunogold electron microscopic imaging. Ultrastructural investigation of mGluR5 expression in S1 cortex was carried out using pre-embedding immunogold electron microscopy as described previously (65), with some modifications. Deeply anesthetized PSL and sham control mice were transcardially perfused with $25 \mathrm{mM}$ PBS (pH 7.4) for 1 minute, followed by perfusion for 12 minutes with $50 \mathrm{ml}$ of a fixative solution of $4 \%$ paraformaldehyde, $0.05 \%$ glutaraldehyde, and $15 \%$ saturated picric acid, all made up in $0.1 \mathrm{M}$ phosphate buffer (PB) (pH 7.4). Coronal sections of S1 cortical region were cut with a microslicer (Linear 7 Pro; Dosaka) at $60 \mu \mathrm{m}$ thickness. These sections were cryoprotected with $30 \%$ sucrose in $\mathrm{PB}$ and freeze thawed with liquid nitrogen several times, blocked for 1 hour in TBS (pH 7.4) containing 10\% normal goat serum (NGS) and then incubated with antimGluR5 guinea pig Ab $(1 \mu \mathrm{g} / \mathrm{ml}$, FRONTIER INSTITUTE Co.) made up in TBS containing $1 \%$ normal goat serum (NGS) overnight at $4^{\circ} \mathrm{C}$. After washing, the sections were incubated with $1.4 \mathrm{~nm}$ gold-coupled anti-guinea pig secondary $\mathrm{Ab}$ (Nanoprobes Inc.) diluted in TBS at a ratio of 1:100 overnight at $4^{\circ} \mathrm{C}$. After washing, the sections were postfixed in $1 \%$ glutaraldehyde for 10 minutes, followed by enhancement of the immunogold signal using a GoldEnhance EM kit (Nanoprobes). Sections were then postfixed with $1 \%$ osmium tetroxide for 40 minutes, en-bloc counterstained with $1 \%$ uranyl acetate for 30 minutes, and dehydrated in graded ethanol series, followed by propylene oxide. The sections were infiltrated overnight at room temperature in Durcupan resin (Sigma-Aldrich) and transferred to glass slides for flat embedding. After resin curing at $60^{\circ} \mathrm{C}$, the trimmed tissues from the ROI (layers I to II/III of S1 on the left hemisphere) were re-embedded in Durcupan resin blocks for ultrathin sectioning. Serial ultrathin sections (70 $\mathrm{nm}$ thickness) were cut within $5 \mu \mathrm{m}$ from the section surface and were collected in pioloform-coated single-slot copper grids. At least 3 ribbons of serial sections containing 10 sections were collected for each group, and serial ultramicroscopic images were captured at well-labeled areas by a CCD camera (Gatan) connected to a JEM1011 transmission electron microscope (JEOL Company).

For individual immunogolds in a randomly selected section within a section ribbon, labeled profiles were judged to be dendrites, spines, presynapses (axons, terminals), or glia (soma, processes) on the basis of the ultrastructural features observed in 2 to 3 neighboring sections for both sides. The frequency of individual immunogolds found in the 4 categories was calculated in each ribbon, and the average and SEM of the frequencies from more than 3 section ribbons were determined for all experimental groups. Labeling specificity for mGluR5 by the Ab was confirmed by pre-embedding immunolabeling with blank staining in mGluR5 KO brain (66).

Combined FISH and IHC. ISH for Tsp1 mRNA was performed using a ViewRNA ISH Tissue kit (Affymetrix) following glial fibrillary acidic protein (GFAP) immunostaining. Briefly, 10\% neutral-buffered formalin fixed $12-\mu \mathrm{m}$ brain cryosections were mounted on glass slides and dehydrated in 50\%, 70\%, and $100 \%$ ethanol each time for 10 minutes at room temperature. The slides were then incubated with protease for 20 minutes at $40^{\circ} \mathrm{C}$ and fixed in $10 \%$ neutral-buffered formalin for 5 minutes at room temperature. Hybridization was carried out with a Tsp1 mRNA-targeting probe set (VB1-16195; Affymetrix) for 3 hours at $40^{\circ} \mathrm{C}$. After signal amplification, tissues were incubated in fast red solution. Then, slides were incubated overnight with antiGFAP primary Ab (1:500; catalog MAB3402; EMD Millipore) and then washed 3 times and further incubated for 1 hour at room temperature with Alexa Fluor 488-conjugated secondary Ab (1:200; Invitrogen) for astrocyte fluorescence staining. Mounting medium with DAPI (VECTASHIELD; Vector Laboratories) was used for covering the slides. Immunofluorescence images were obtained using a confocal microscope with LSM5 PASCAL (Zeiss).

Statistics. Data are presented as mean \pm SEM. Statistical tests were performed using a 2-tailed, unpaired $t$ test (2 variables) or 1-way ANOVA, followed by a Dunnett's multiple comparisons test ( $\geq 3$ variables). If the data did not meet the assumption of normal distribution, nonparametric tests were used, including the Mann-Whitney $U$ (2 variables) or Kruskal-Wallis test, followed by a Dunn's multiple comparisons test ( $\geq 3$ variables). In all cases, a $P$ value of less than 0.05 was considered statistically significant. Sample sizes were determined by considering those typically used in similar studies as well as by the variation and mean of the samples. No specific randomization methods were used to assign animals to the experimental groups. We excluded animals from the analysis when they showed any abnormality (e.g., poor general condition, small body size, low body weight, brain damage during the craniotomy) prior to or during the experiments.

Study approval. All animal experiments were approved by the Animal Research Committee of the National Institute for Physiological Sciences and the Animal Care Committee of the University of Yamanashi. Every effort was made to minimize the number of animals used and their suffering.

\section{Author contributions}

S.KK, SK, and JN conceived and designed the study. SKK, HH, TI, KS, ES, YS, HI, SER, SJK, GL, HB, AJM, and YF performed the experiments. KM provided $\mathrm{IP}_{3} \mathrm{R} 2-\mathrm{KO}$ mice and their littermates. SKK, AJM, SK, and JN analyzed and interpreted the data and wrote the manuscript. All of the authors read and discussed the manuscript.

\section{Acknowledgments}

We thank M. Kano for the gift of Elvax beads; D.X. Li, H. Yoon, S.H. Kim, K. Nakamura, T. Toda, R. Akiyoshi, A. Miyamoto, K. Eto, H. Wake, and H. Ishibashi for excellent technical assistance and critical discussions on the experiments. This work was supported by a Core Research for Evolutional Science and Technology (CREST) grant from the Japan Agency for Medical Research and Development (AMED) and from the Japan Science and Technology Agency (JST) (to J. Nabekura); Grant-in-Aids for Scientific 
Research (A) (22240042, to J. Nabekura) and (S) (25221002, to K. Mikoshiba) from the Japan Society for the Promotion of Science (JSPS); a Grant-in-Aid for Scientific Research on an Innovative Area from the Ministry of Education, Culture, Sports, Science and Technology (MEXT) of Japan (to S. Koizumi); and by grants from the National Research Foundation of Korea funded by the Korean government (NRF-2013R1A1A1012403 and MEST-20120005755, to S.K. Kim and MSIP-2011-0030737, to S.J. Kim). The funders had no role in study design, data collection and analysis, decision to publish, or preparation of the manuscript.

Address correspondence to: Junichi Nabekura, 38 Nishigonaka Myodaiji, Okazaki, Aichi 444-8585, Japan. Phone: 81.564.55.7851; E-mail: nabekura@nips.ac.jp. Or to: Schuichi Koizumi, 1110 Shimokato, Chuo, Yamanashi 409-3898, Japan. Phone: 81.55.273. 9503; E-mail: skoizumi@yamanashi.ac.jp.
1. Apkarian AV, Baliki MN, Geha PY. Towards a theory of chronic pain. Prog Neurobiol. 2009;87(2):81-97.

2. Zhuo M. Cortical excitation and chronic pain. Trends Neurosci. 2008;31(4):199-207.

3. Fu M, Zuo Y. Experience-dependent structural plasticity in the cortex. Trends Neurosci. 2011;34(4):177-187.

4. Holtmaat A, Svoboda K. Experience-dependent structural synaptic plasticity in the mammalian brain. Nat Rev Neurosci. 2009;10(9):647-658.

5 . Baliki MN, et al. Corticostriatal functional connectivity predicts transition to chronic back pain. Nat Neurosci. 2012;15(8):1117-1119.

6. Metz AE, Yau HJ, Centeno MV, Apkarian AV, Martina M. Morphological and functional reorganization of rat medial prefrontal cortex in neuropathic pain. Proc Natl Acad Sci U S A. 2009;106(7):2423-2428.

7. Koga K, et al. Coexistence of two forms of LTP in ACC provides a synaptic mechanism for the interactions between anxiety and chronic pain. Neuron. 2015;85(2):377-389.

8. Li XY, et al. Alleviating neuropathic pain hypersensitivity by inhibiting PKMzeta in the anterior cingulate cortex. Science. 2010;330(6009):1400-1404.

9. Eto K, et al. Inter-regional contribution of enhanced activity of the primary somatosensory cortex to the anterior cingulate cortex accelerates chronic pain behavior. J Neurosci. 2011;31(21):7631-7636.

10. Kim SK, Nabekura J. Rapid synaptic remodeling in the adult somatosensory cortex following peripheral nerve injury and its association with neuropathic pain. J Neurosci. 2011;31(14):5477-5482.

11. Bushnell MC, Duncan GH, Hofbauer RK, Ha B, Chen JI, Carrier B. Pain perception: is there a role for primary somatosensory cortex? Proc Natl Acad Sci U S A. 1999;96(14):7705-7709.

12. Gross J, Schnitzler A, Timmermann L, Ploner M. Gamma oscillations in human primary somatosensory cortex reflect pain perception. PLOS Biol. 2007;5(5):e133.

13. Monconduit L, Lopez-Avila A, Molat JL, Chalus M, Villanueva L. Corticofugal output from the primary somatosensory cortex selectively modulates innocuous and noxious inputs in the rat spinothalamic system. J Neurosci. 2006;26(33):8441-8450.

14. De Ridder D, De Mulder G, Menovsky T, Sunaert $\mathrm{S}$, Kovacs S. Electrical stimulation of auditory and somatosensory cortices for treatment of tinnitus and pain. Prog Brain Res. 2007;166:377-388.

15. Flor H, Nikolajsen L, Staehelin Jensen T. Phantom limb pain: a case of maladaptive CNS plas- ticity? Nat Rev Neurosci. 2006;7(11):873-881.

16. Matsuzawa-Yanagida K, et al. Usefulness of antidepressants for improving the neuropathic pain-like state and pain-induced anxiety through actions at different brain sites. Neuropsychopharmacology. 2008;33(8):1952-1965.

17. Kim SK, Kato G, Ishikawa T, Nabekura J. Phase-specific plasticity of synaptic structures in the somatosensory cortex of living mice during neuropathic pain. Mol Pain. 2011;7:87.

18. Bhatt DH, Zhang S, Gan WB. Dendritic spine dynamics. Annu Rev Physiol. 2009;71:261-282.

19. Kim SK, Eto K, Nabekura J. Synaptic structure and function in the mouse somatosensory cortex during chronic pain: in vivo two-photon imaging. Neural Plast. 2012;2012:640259.

20. Yi M, Zhang H. Nociceptive memory in the brain: cortical mechanisms of chronic pain. J Neurosci. 2011;31(38):13343-13345.

21. Agulhon C, et al. What is the role of astrocyte calcium in neurophysiology? Neuron. 2008;59(6):932-946.

22. Volterra A, Meldolesi J. Astrocytes, from brain glue to communication elements: the revolution continues. Nat Rev Neurosci. 2005;6(8):626-640.

23. Eroglu C, Barres BA. Regulation of synaptic connectivity by glia. Nature. 2010;468(7321):223-231

24. Ippolito DM, Eroglu C. Quantifying synapses: an immunocytochemistry-based assay to quantify synapse number. J Vis Exp. 2010;(45):2270.

25. Futatsugi A, et al. IP3 receptor types 2 and 3 mediate exocrine secretion underlying energy metabolism. Science. 2005;309(5744):2232-2234.

26. Agulhon C, Fiacco TA, McCarthy KD. Hippocampal short- and long-term plasticity are not modulated by astrocyte $\mathrm{Ca}^{2+}$ signaling. Science. 2010;327(5970):1250-1254.

27. Sharp AH, et al. Differential cellular expression of isoforms of inositol 1,4,5-triphosphate receptors in neurons and glia in brain. JComp Neurol. 1999;406(2):207-220.

28. Takata N, et al. Astrocyte calcium signaling trans forms cholinergic modulation to cortical plasticity in vivo. J Neurosci. 2011;31(49):18155-18165.

29. Ding S, et al. Enhanced astrocytic $\mathrm{Ca}^{2+}$ signals contribute to neuronal excitotoxicity after status epilepticus. J Neurosci. 2007;27(40):10674-10684.

30. Takano T, et al. Astrocyte-mediated control of cerebral blood flow. Nat Neurosci. 2006;9(2):260-267.

31. Feng G, et al. Imaging neuronal subsets in transgenic mice expressing multiple spectral variants of GFP. Neuron. 2000;28(1):41-51.

32. Eroglu C, et al. Gabapentin receptor alpha2 delta- 1 is a neuronal thrombospondin receptor responsible for excitatory CNS synaptogenesis.
Cell. 2009;139(2):380-392.

33. Liauw J, et al. Thrombospondins 1 and 2 are necessary for synaptic plasticity and functional recovery after stroke. J Cereb Blood Flow Metab. 2008;28(10):1722-1732.

34. Christopherson KS, et al. Thrombospondins are astrocyte-secreted proteins that promote CNS synaptogenesis. Cell. 2005;120(3):421-433.

35. Parkhurst CN, et al. Microglia promote learning-dependent synapse formation through brain-derived neurotrophic factor. Cell. 2013;155(7):1596-1609.

36. Zhang F, Vadakkan KI, Kim SS, Wu LJ, Shang Y, Zhuo M. Selective activation of microglia in spinal cord but not higher cortical regions following nerve injury in adult mouse. Mol Pain. 2008;4:15.

37. Costigan M, Scholz J, Woolf CJ. Neuropathic pain: a maladaptive response of the nervous system to damage. Annu Rev Neurosci. 2009;32:1-32.

38. Melzack R, Coderre TJ, Katz J, Vaccarino AL. Central neuroplasticity and pathological pain. Ann N Y Acad Sci. 2001;933:157-174.

39. Sun W, et al. Glutamate-dependent neuroglial calcium signaling differs between young and adult brain. Science. 2013;339(6116):197-200.

40. DaSilva AF, Becerra L, Pendse G, Chizh B, Tully S, Borsook D. Colocalized structural and functional changes in the cortex of patients with trigeminal neuropathic pain. PLoS One. 2008;3(10):e3396.

41. Gustin SM, Peck CC, Cheney LB, Macey PM, Murray GM, Henderson LA. Pain and plasticity: is chronic pain always associated with somatosensory cortex activity and reorganization? J Neurosci. 2012;32(43):14874-14884.

42. Peyron R, et al. An fMRI study of cortical representation of mechanical allodynia in patients with neuropathic pain. Neurology. 2004;63(10):1838-1846.

43. Cauda F, et al. Low-frequency BOLD fluctuations demonstrate altered thalamocortical connectivity in diabetic neuropathic pain. BMC Neurosci. 2009;10:138.

44. Kim CE, et al. Identifying neuropathic pain using (18)F-FDG micro-PET: a multivariate pattern analysis. NeuroImage. 2014;86:311-316.

45. Inoue K, Koizumi S, Tsuda M. The role of nucleotides in the neuron--glia communication responsible for the brain functions. J Neurochem. 2007;102(5):1447-1458.

46. McMahon SB, Malcangio M. Current challenges in glia-pain biology. Neuron. 2009;64(1):46-54.

47. Chklovskii DB, Mel BW, Svoboda K. Cortical rewiring and information storage. Nature. 2004;431(7010):782-788.

48. Xu H, et al. Presynaptic and postsynaptic amplifi- 
cations of neuropathic pain in the anterior cingulate cortex. JNeurosci. 2008;28(29):7445-7453.

49. Fields RD, Stevens-Graham B. New insights into neuron-glia communication. Science. 2002;298(5593):556-562.

50. Kim DS, et al. Thrombospondin- 4 contributes to spinal sensitization and neuropathic pain states. J Neurosci. 2012;32(26):8977-8987.

51. Araque A, Carmignoto G, Haydon PG, Oliet SH, Robitaille R, Volterra A. Gliotransmitters travel in time and space. Neuron. 2014;81(4):728-739.

52. Bernardinelli Y, et al. Activity-dependent structural plasticity of perisynaptic astrocytic domains promotes excitatory synapse stability. Curr Biol. 2014;24(15):1679-1688.

53. Nedergaard M, Verkhratsky A. Artifact versus reality - how astrocytes contribute to synaptic events. Glia. 2012;60(7):1013-1023.

54. Panatier A, Vallee J, Haber M, Murai KK, Lacaille JC, Robitaille R. Astrocytes are endogenous regulators of basal transmission at central synapses. Cell. 2011;146(5):785-798.

55. Tyzack GE, et al. Astrocyte response to motor neuron injury promotes structural synaptic plas- ticity via STAT3-regulated TSP-1 expression. Nat Commun. 2014;5:4294.

56. Garcia O, Torres M, Helguera P, Coskun P, Busciglio J. A role for thrombospondin-1 deficits in astrocyte-mediated spine and synaptic pathology in Down's syndrome. PLoS One. 2010;5(12):e14200.

57. Malmberg AB, Basbaum AI. Partial sciatic nerve injury in the mouse as a model of neuropathic pain: behavioral and neuroanatomical correlates. Pain. 1998;76(1-2):215-222.

58. Chaplan SR, Bach FW, Pogrel JW, Chung JM, Yaksh TL. Quantitative assessment of tactile allodynia in the rat paw. JNeurosci Methods. 1994;53(1):55-63.

59. Shibata K, et al. The astrocyte-targeted therapy by Bushi for the neuropathic pain in mice. PLoS One. 2011;6(8):e23510.

60. Holtmaat A, et al. Long-term, high-resolution imaging in the mouse neocortex through a chronic cranial window. Nat Protoc. 2009;4(8):1128-1144.

61. Stosiek C, Garaschuk O, Holthoff K, Konnerth A. In vivo two-photon calcium imaging of neuronal networks. Proc Natl Acad Sci U S A. 2003;100(12):7319-7324.
62. Kakizawa S, Miyazaki T, Yanagihara D, Iino M, Watanabe M, Kano M. Maintenance of presynaptic function by AMPA receptor-mediated excitatory postsynaptic activity in adult brain. Proc Natl Acad Sci U S A. 2005;102(52):19180-19185.

63. Hayakawa K, Pham LD, Katusic ZS, Arai K, Lo EH. Astrocytic high-mobility group box 1 promotes endothelial progenitor cell-mediated neurovascular remodeling during stroke recovery. Proc Natl Acad Sci U S A. 2012;109(19):7505-7510.

64. Koizumi S, Fujishita K, Tsuda M, ShigemotoMogami Y, Inoue K. Dynamic inhibition of excitatory synaptic transmission by astrocytederived ATP in hippocampal cultures. Proc Natl Acad Sci U S A. 2003;100(19):11023-11028.

65. Parajuli LK, et al. Quantitative regional and ultrastructural localization of the $\mathrm{Ca}(\mathrm{v}) 2.3$ subunit of R-type calcium channel in mouse brain. J Neurosci. 2012;32(39):13555-13567.

66. Tanaka J, et al. Gq protein alpha subunits Galphaq and Galpha11 are localized at postsynaptic extrajunctional membrane of cerebellar Purkinje cells and hippocampal pyramidal cells. Eur J Neurosii. 2000;12(3):781-792. 